\title{
Exploiting Resolution Proofs to Speed Up LTL Vacuity Detection for BMC
}

\author{
Jocelyn Simmonds ${ }^{1}$, Jessica Davies ${ }^{1}$, Arie Gurfinkel ${ }^{2}$, Marsha Chechik $^{1}$ \\ 1 Department of Computer Science, University of Toronto \\ 2 Software Engineering Institute, Carnegie Mellon University
}

The date of receipt and acceptance will be inserted by the editor

\begin{abstract}
When model-checking reports that a property holds on a model, vacuity detection increases user confidence in this result by checking that the property is satisfied in the intended way. While vacuity detection is effective, it is a relatively expensive technique requiring many additional modelchecking runs. We address the problem of efficient vacuity detection for Bounded Model Checking (BMC) of LTL properties, presenting three partial vacuity detection methods based on the efficient analysis of the resolution proof produced by a successful BMC run. In particular, we define a characteristic of resolution proofs - peripherality - and prove that if a variable is a source of vacuity, then there exists a resolution proof in which this variable is peripheral. Our vacuity detection tool, VaqTree, uses these methods to detect vacuous variables, decreasing the total number of model-checking runs required to detect all sources of vacuity.
\end{abstract}

\section{Introduction}

Model-checking [7] is a widely-used automated technique for verification of both hardware and software artifacts that checks whether a temporal logic property is satisfied by a finite-state model of the artifact. If the model does not satisfy the property, a counterexample, which can aid in debugging, is produced. If the model does satisfy the property, no information about why it does so is provided by the model-checker alone. A positive answer without any additional information can be misleading, since a property may be satisfied in a way that was not intended. For instance, a property "every request is eventually acknowledged" is satisfied in an environment that never generates requests.

Vacuity detection $[2,18,21,1]$ is an automatic sanity check that can be applied after a positive model-checking run in order to gain confidence that the model and the property capture the desired behaviours. Informally, a property is said to be vacuous if it has a subformula which is not relevant to its satisfaction, or if the property itself is a tautology. Conversely, a property is satisfied non-vacuously if every part of the formula is important - even a slight change to the formula affects its satisfaction.

In this article, we focus on vacuity detection for SATbased Bounded Model Checking (BMC). Given a BMC problem with a particular bound, we wish to determine if the property holds vacuously on the model up to this bound. In this context, a naive method for detecting vacuity is to replace subformulas of the temporal logic property with unconstrained boolean variables and run BMC for each such substitution. If the property with some substitution still holds on the model, the property is vacuous. This naive approach is expensive, since in the worst case it requires as many modelchecking runs as there are subformulas in the property. Our goal is to reduce the number of model-checking runs required to detect vacuity. We do this by detecting some vacuity through novel and inexpensive techniques reported in this article, and complete the method by running the naive algorithm on the remaining atomic subformulas. The key to our technique is that SAT-based BMC can automatically provide useful information (a resolution proof) beyond a decision whether the property holds on the model; we exploit such proofs for partial vacuity detection.

In SAT-based BMC, the property and the behavior of the model are encoded in a propositional theory, such that the theory is satisfiable if and only if the formula does not hold. When the property does hold, a DPLL-based SAT solver can produce a resolution proof that derives false from a subset of the clauses in the theory called the UNSAT core. Intuitively, the resolution proof provides an explanation of why the property is not falsified by the model, and the UNSAT core determines the relevant parts of the model and the property [19].

In this article, we develop three methods of increasing precision (irrelevance, local irrelevance, and peripherality) to analyze the resolution proof to achieve partial vacuity detection. These algorithms are used by our vacuity detection 
tool, VaqTree, in order to reduce the number of model-checking runs required to find all sources of vacuity, thus reducing execution times. Irrelevance and local irrelevance detect vacuity based on which variables appear in the UNSAT core, and in which locations. However, as these methods only examine the UNSAT core, their precision is limited. The peripherality algorithm examines the structure of the resolution proof, identifying as vacuous those variables that are not necessary or central to the derivation of false. This method is as precise as can be achieved through analyzing a single resolution proof, and its running time is linear in the size of the resolution proof and the number of variables in the property. Our experience shows that local irrelevance is the ideal candidate for speeding up naive vacuity detection.

The remainder of the article is organized as follows. Section 2 presents some required background, followed, in Section 3 by our definition of vacuity, the naive algorithm for LTL vacuity detection using BMC, and an overview of work in the vacuity detection field. Section 4 presents the three algorithms that detect vacuity by analyzing a resolution proof. Tool support for our approach is described in Section 5. Our experimental results are presented in Section 6. We conclude with a summary, additional related work, and suggestions for future work in Section 7.

\section{Background}

In this section, we review bounded model-checking and resolution proofs.

\subsection{Bounded Model-Checking}

Bounded model-checking (BMC) [4] is a method for determining whether a linear temporal logic (LTL) formula $\varphi$ holds on a finite state system represented by a Kripke structure $K$ up to a finite number of steps. To solve an instance of the BMC problem, denoted by $B M C_{k}(K, \varphi)$, it is required to determine whether $K \models_{k} \varphi$, where $\models_{k}$ is the $k$-depth satisfaction relation. Below, we give an informal overview of Kripke structures, LTL formulas and BMC. More detailed definitions can be found in $[7,4]$.

A Kripke structure $K$ has a finite set of states $S$, one of which is considered to be the initial state $s_{0}$. A transition relation $R \subseteq S \times S$ relates states to states. Each state is labeled by the set of propositional formulas (or variables) that hold in that state. A run of $K$ is a sequence of states starting with $s_{0}$ that obeys $R$. Each run has an associated trace $\pi$, where $\pi_{i}$ is simply the set of propositional formulas that label the $i^{t h}$ state in the run. We write $\pi^{i}$ to denote the suffix of the trace beginning at $i$.

LTL formulas are built from propositional variables, the usual boolean operators $(\vee, \wedge, \neg)$, and the temporal operators $\mathbf{G}$ ("always"), $\mathbf{F}$ ("eventually"), $\mathbf{U}$ ("until"), and $\mathbf{X}$ ("next"). Their semantics are defined on linear traces, such as those produced by runs of a Kripke structure. $\pi \models \varphi$ means

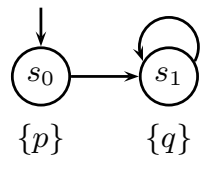

Fig. 1: A Kripke structure.

that the trace $\pi$ satisfies the LTL formula $\varphi$. For example, $\pi \models \mathbf{F} \varphi$ if and only if there exists some $i$ such that $\varphi$ holds on $\pi^{i}$. The satisfaction relation $\models$ is defined inductively in a similar way for all operators and propositional variables in LTL. We refer the reader to [7] for a detailed description of the semantics of LTL.

A Kripke structure $K$ satisfies an LTL formula $\varphi$ if and only if $\pi \models \varphi$ for all traces $\pi$ of $K$. The BMC problem $B_{M C} C_{k}(K, \varphi)$ is to determine whether $K$ satisfies $\varphi$ for up to $k$ steps, i.e., whether $K \models_{k} \varphi$. The $k$-depth satisfaction relation $\models_{k}$ is defined inductively; for example, $\pi \models_{k} \mathbf{G} \varphi$ if and only if $\pi^{i} \models_{k-1} \varphi$ for all $i \leq k$.

To determine whether $K \models_{k} \varphi$, the problem is converted to a propositional formula $\Phi$ (see $[4,6,5]$ ) which is satisfiable if and only if there exists a length- $k$ counterexample to $K \models_{k} \varphi . \Phi$ is then given to a SAT solver which decides its satisfiability. The propositional encoding represents the behavior of $K$ up to $k$ steps with a path constraint $C L_{K}$, and encodes all counterexamples to $\varphi$ of length $k$ in an error constraint $C L_{e}$. Therefore, if the theory $C L_{K} \cup C L_{e}$ is satisfiable, there is a path through $K$ which obeys the transition relation and falsifies $\varphi$. The value of each variable $v$ of $K$ at each time step is represented using new boolean variables $v_{i}$ $(0 \leq i \leq k)$, called timed variables.

The transition relation of a Kripke structure can be represented symbolically by a propositional formula over the variables $V$ and primed variables $V^{\prime}$ (which represent the variables in the next state). For example, in the model in Figure 1 , the transition relation is represented by the formula $R=\left(p \wedge \neg q \wedge \neg p^{\prime} \wedge q^{\prime}\right) \vee\left(\neg p \wedge q \wedge \neg p^{\prime} \wedge q^{\prime}\right)$. The path constraint is obtained by substituting the timed variables $V_{i}$ for $V$ in $R$, and replacing $V^{\prime}$ by the timed variables for the next step, $V_{i+1}$. This is repeated for each $0 \leq i<k$, and the resulting propositional formulas are conjoined along with a formula representing the initial state [4]. In Figure 1, if $k=1$,

$$
\begin{gathered}
C L_{K}=\left(p_{0} \wedge \neg q_{0}\right) \wedge\left(\left(p_{0} \wedge \neg q_{0} \wedge \neg p_{1} \wedge q_{1}\right)\right. \\
\left.\vee\left(\neg p_{0} \wedge q_{0} \wedge \neg p_{1} \wedge q_{1}\right)\right) .
\end{gathered}
$$

The error constraint $C L_{e}$ is encoded according to a recursive procedure which removes the temporal and logical operators from the property [4], e.g., the algorithm encodes $\varphi=\mathbf{G} p$, where $p$ is a propositional variable, expanded up to $k=2$, by the formula $\neg p_{0} \vee \neg p_{1} \vee \neg p_{2}$.

After the boolean formulas for the path and error constraints are calculated, they are converted to Conjunctive Normal Form (CNF) before being passed to a SAT solver. If the solver reports that $C L_{K} \cup C L_{e}$ is unsatisfiable, it means that there is no length- $k$ counterexample to $\varphi$; otherwise, a 


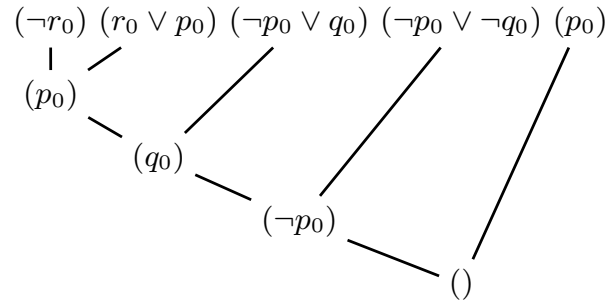

Fig. 2: A resolution proof for EXAMPLE 2.

satisfying assignment is returned. When a DPLL-based SAT solver processes an unsatisfiable theory, a resolution derivation of false (or the empty clause) is implicitly constructed [10, 27]. This resolution proof is used to verify that false can indeed be derived from $C L_{K} \cup C L_{e}$ [28].

\subsection{Resolution Proofs}

Resolution is an inference rule that is applied to propositional clauses to produce logical consequences. A clause is a disjunction of literals (boolean variables or their negations). For example, $\left(v_{1} \vee \neg v_{2} \vee v_{5}\right)$ is a clause stating that at least one of $v_{1}, \neg v_{2}$ or $v_{5}$ must be true. The resolution rule takes two clauses, where one contains a literal $v$ and the other - its negation $\neg v$, and produces a clause containing the union of the two clauses' literals minus $v$ and $\neg v$. For example, resolving $\left(v_{1} \vee \neg v_{2} \vee v_{5}\right)$ and $\left(v_{2} \vee v_{6}\right)$ produces the resolvent $\left(v_{1} \vee v_{5} \vee v_{6}\right)$.

A resolution proof $\Pi$ is a directed acyclic graph whose nodes are labeled by propositional clauses. $\Pi$ represents a tree of resolutions between the clauses labeling its nodes. Its roots are the nodes with no parents; otherwise, all nodes have exactly two parents. The nodes with no children are called the leaves. For example, the roots of resolution proof $\Pi$ in Figure 2 are Roots $(\Pi)=\left\{\left(\neg r_{0}\right),\left(r_{0} \vee p_{0}\right),\left(\neg p_{0} \vee\right.\right.$ $\left.\left.q_{0}\right),\left(\neg p_{0} \vee \neg q_{0}\right),\left(p_{0}\right)\right\}$, and the leaf of $\Pi$ is the empty clause, i.e., Leaf $(\Pi)=$ false. Given a non-root node labeled by the clause $c$, and the labels of its parents, $c_{1}$ and $c_{2}, c$ is the resolvent since it has been produced by resolving $c_{1}$ and $c_{2}$ on some variable $v$. A resolution proof $\Pi$ is a proof of unsatisfiability of a set of clauses $A$ if and only if all roots of $\Pi$ belong to $A$, and one of the leaves of $\Pi$ is the empty clause. For example, Figure 2 shows a resolution proof of the unsatisfiability of Roots $(\Pi)$. If a propositional theory in CNF is unsatisfiable, an UNSAT core is an unsatisfiable subset of its clauses.

Given two disjoint sets of clauses $A$ and $B$, a variable $v$ is said to be local to $A$ if and only if $v$ appears in $A$ but does not appear in $B$, and $v$ is said to be global if it appears in both $A$ and $B$. In Figure 2, if $\operatorname{Roots}(\Pi)=A \cup B$, where $A=$ $\left\{\left(\neg r_{0}\right),\left(r_{0} \vee p_{0}\right),\left(\neg p_{0} \vee q_{0}\right)\right\}$ and $B=\left\{\left(\neg p_{0} \vee \neg q_{0}\right),\left(p_{0}\right)\right\}$, then $r_{0}$ is local to $A$, and the rest of the variables are global.

\section{Defining Vacuity}

This article uses the following definition of vacuity.

Definition 1. Let $K$ be a Kripke structure, $\varphi$ be a formula s.t. $K \models_{k} \varphi$, and $p$ be a variable. $\varphi$ is $k$-step p-vacuous iff $K \models_{k} \varphi[p \leftarrow x]$, where $x$ is a variable not occurring in $K$ or in $\varphi$.

If $\varphi$ is $k$-step $p$-vacuous, we call $p$ a $k$-step vacuous variable. A property $\varphi$ is $k$-step vacuous if and only if $\varphi$ contains a $k$-step vacuous variable. Therefore, our techniques aim to find the $k$-step vacuous variables of $\varphi$. The qualifier " $k$-step" is omitted in the remainder of the article but should be understood implicitly in the BMC context.

Definition 1 can be generalized to vacuity in arbitrary (not necessarily atomic) subformulas. This follows from the fact that a subformula is vacuous iff it is mutually vacuous in all of its atomic propositions [13, Th. 9], and that the definitions can be easily extended to mutual vacuity. A set of atomic propositions $\left\{p_{1}, \ldots, p_{n}\right\}$ is mutually vacuous if $K \models_{k} \varphi\left[p_{1} \leftarrow\right.$ $\left.x_{1}, \ldots, p_{n} \leftarrow x_{n}\right]$, where $\left\{x_{1}, \ldots, x_{n}\right\}$ are new variables. For example, if $\varphi$ contains subformula $\theta=p \wedge q$, and $p$ and $q$ are mutually vacuous, then we can deduce that $\theta$ is vacuous as well.

Naive Vacuity Detection. Definition 1 suggests a sound and complete algorithm for vacuity detection: for each propositional variable $p$ in $\varphi$, run BMC on $\varphi[p \leftarrow x]$, where $x$ is a variable that does not appear in $K$ and $\varphi$. If $K \models_{k} \varphi[p \leftarrow x]$ for some $p$, then $\varphi$ is $k$-step vacuous. We refer to this algorithm as naive. Its drawback is that it may require as many model-checking runs as there are propositional variables in $\varphi$.

We now review some of the alternative definitions of vacuity and their algorithms. The first attempt to formulate and automate vacuity detection is due to Beer et al. [2]. They consider a property $\varphi$ to be vacuous if $\varphi$ contains a subformula $\psi$ such that replacing $\psi$ by any other formula does not affect the satisfaction of $\varphi$. Applying this definition directly would require an infinite number of subformula replacements, precluding a practical implementation. However, Beer et al. show that to detect vacuity w.r.t. a single occurrence of a subformula $\psi$ in w-ACTL, it is sufficient to replace $\psi$ with only true and false. This was later extended to CTL* by Kupferman and Vardi [18], and to the modal $\mu$-calculus by Dong et al. [9]. Purandare and Somenzi [21] showed how to speed up subformula vacuity by analyzing the parse tree of a CTL property.

Armoni et al. [1] generalized the above syntactic definition of vacuity by introducing universal quantification, i.e., $\forall x \cdot \varphi[\psi \leftarrow x]$. Based on the domain of $x$, three notions of vacuity are obtained, the most robust of which being trace vacuity. Gurfinkel and Chechik [12] extended Armoni's definition of vacuity to CTL*, thus uniformly capturing CTL and LTL. Armoni et al. also analyzed the syntactic structure of the property in order to avoid checking the operands of subformulas that are known to be vacuous. Such optimizations 
complement our techniques, which focus on detecting vacuous atomic subformulas.

In [20], Namjoshi has introduced a proof-based variant of vacuity. Although it is called proof vacuity in the original paper, we refer to it as forall-proof vacuity. This definition is based on the semantic proofs of $K \models \varphi$ for a Kripke structure $K$ and a formula $\varphi$. Informally, a formula $\varphi$ is forall-proof vacuous in a subformula $\psi$ if $\psi$ is not used in any proof of $K \models \varphi$. Of course, a formal definition depends on the exact interpretation of the notion of "proof". In comparison, we use an "existential" definition of vacuity: a formula is vacuous if there exists a proof that does not use a subformula. Interestingly, we rely on syntactic (and not semantic) resolution proofs that may include "semantically-useless" resolutions. As a result, it is possible that a formula $\varphi$ is vacuous in $\psi$ in a model $K$, yet there is no resolution proof of bounded satisfaction of $K \models \varphi$ that does not use $\psi$. More importantly, our goal is to develop a method to efficiently detect vacuity for LTL as it was defined by $[2,3,1,12]$, whereas Namjoshi was looking for an alternative definition of vacuity for branching time logic.

Our definition of vacuity is syntactic, and in this respect, it is similar to the original definition of Beer et al. [2]. However, Definition 1 is stronger, and is equivalent to the semantic definition of Armoni et al. [1], as shown by Gurfinkel and Chechik [12].

\section{Exploiting Resolution Proofs}

In Section 3, we discussed the existence of a sound and complete vacuity detection algorithm for BMC, which requires as many model-checking runs as there are propositional variables in the property being checked. We propose a new vacuity detection strategy: first detect partial vacuity using inexpensive techniques and then complete the analysis using extra model-checking runs. Since we are interested in replacing expensive model-checking runs by inexpensive partial vacuity detection methods, we limit ourselves to considering the output of the original model-checking run on $B M C_{k}(K, \varphi)$, i.e., $C L_{K} \cup C L_{e}$. This run provides us with a single resolution proof to analyze. Of course, in general, there may be many ways to derive the empty clause from different subsets of $B M C_{k}(K, \varphi)$. Any method that only examines one of these derivations is inherently incomplete, in the sense that a property may be $p$-vacuous but there is no way of determining this based on a given resolution proof. For example, consider a model that is composed of two completely disjoint sub-models, running in parallel, i.e., $K=K_{1} \| K_{2}$. Suppose that $K_{1}$ satisfies $\mathbf{G} p, K_{2}$ satisfies $\mathbf{G} q$, and that both do so non-vacuously. Then the property $\varphi=\mathbf{G} p \vee \mathbf{G} q$ holds on $K$ $p$-vacuously and $q$-vacuously. However, one of the possible resolution proofs showing that $\varphi$ holds proves that $\mathbf{G} p$ holds non-vacuously on $K_{1}$. Thus, it is impossible to determine that $\varphi$ is vacuous in $p$ from this proof. Any method based on examining only one resolution proof cannot prove the absence of vacuity, since another resolution proof, showing the property to be vacuous, might exist.

In this section, we introduce three algorithms of increasing precision for partial vacuity detection, based on examining the UNSAT core (irrelevance and local irrelevance) and the resolution proof produced by BMC (peripherality).

\subsection{Examining UNSAT cores}

Given a resolution proof that $B M C_{k}(K, \varphi)$ is unsatisfiable, we can sometimes cheaply determine that the similar theory $B M C_{k}(K, \varphi[p \leftarrow x])$ is also unsatisfiable, and therefore, that the property is $p$-vacuous. In this section, we consider how to determine that $B M C_{k}(K, \varphi[p \leftarrow x])$ is unsatisfiable given that $B M C_{k}(K, \varphi)$ is unsatisfiable, using only an UNSAT core.

\subsubsection{Irrelevance}

Intuitively, any variable that does not appear in the UNSAT core does not contribute to the reason why $\varphi$ holds on $K$, so it can be considered irrelevant.

Definition 2. Let $K$ be a model, and $\varphi$ an LTL formula. Assume that $\Pi$ is an UNSAT core of $\operatorname{BMC}_{k}(\mathrm{~K}, \varphi)$ witnessing that $K \models_{k} \varphi$. Then, $p$ is irrelevant with respect to $\mathrm{BMC}_{k}(\mathrm{~K}, \varphi)$ and $\Pi$ iff $p_{i}$ does not appear in $\Pi$ for any time instance $i$.

If a variable is irrelevant, it is also vacuous, as shown by the following theorem.

Theorem 1. If $p$ is irrelevant with respect to $B M C_{k}(K, \varphi)$ and $\Pi$, then $\varphi$ is $k$-step p-vacuous.

Proof: Let $B M C_{k}(K, \varphi)=C L_{K} \cup C L_{e}$ and $U$ be the UNSAT core returned by the SAT solver for $B M C_{k}(K, \varphi)$. Assume that $p$ is irrelevant in $B M C_{k}(K, \varphi)$. So $U$ does not contain any $p_{i}$ by Definition 2. Therefore, $U \subseteq C L_{K} \cup C L_{e}$ implies $U \subseteq C L_{K} \cup$ $C L_{e}\left[p_{i} \leftarrow x_{i} \mid 0 \leq i<k\right] . U$ is also an UNSAT core of $B M C_{k}(K, \varphi[p \leftarrow x])$ so $\varphi[p \leftarrow x]$ holds on $K$. Thus, $\varphi$ is $p$ vacuous.

Definition 2 provides an algorithm to detect some vacuous variables. However, a variable can appear in the UNSAT core and still be vacuous, as demonstrated by the following example.

EXAMPLE 1. Consider a Kripke structure $K$ with variables $p$ and $q$ given by the constraints Init $=p \wedge q, R=p \Rightarrow q^{\prime}$, which mean that the initial state is labeled by $\{p, q\}$, and the transition relation is expressed by the propositional formula $p \Rightarrow q^{\prime}$ over unprimed and primed variables. Let $\varphi=$ $X(p \vee q)$ be the property to check. $\varphi$ is $p$-vacuous since it is satisfied simply because $q$ is true in any successor of the initial state. The CNF encoding of the one-step BMC problem is $C L_{K}=\left\{\left(p_{0} \wedge q_{0}\right),\left(p_{0} \Rightarrow q_{1}\right)\right\}=\left\{\left(p_{0}\right),\left(q_{0}\right),\left(\neg p_{0}, q_{1}\right)\right\}$, $C L_{e}=\left\{\left(\neg p_{1}\right),\left(p_{1}, \neg q_{1}\right)\right\}$. In this case, the unique minimal UNSAT core contains all of the clauses of the problem except for $\left(q_{0}\right)$. Thus, all $p_{i}$ appear in the UNSAT core, and $p$ cannot be determined vacuous using irrelevance. 


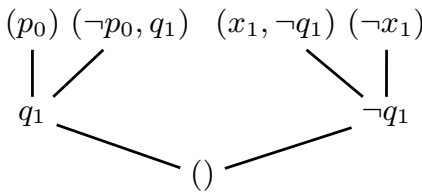

Fig. 3: A resolution proof for EXAMPLE 1.

This example shows that even if we are to look at every UNSAT core of a BMC problem, irrelevance is still unable to detect existing vacuity.

\subsubsection{Local Irrelevance}

Variables which do not appear in the UNSAT core are vacuous. The converse is not true: vacuous variables may also appear in the UNSAT core. Intuitively, these variables are not the central reason why $\varphi$ holds on $K$. For example, the clauses of $C L_{K}$ may resolve against each other, representing some simplification and unification of parts of the model, before resolutions with $C L_{e}$ clauses are performed. If a variable is resolved upon using only the $C L_{K}$ clauses or only the $C L_{e}$ clauses, it is potentially vacuous. By looking at the UNSAT core, it is possible to anticipate whether a variable will not be involved in resolutions between $C L_{K}$ and $C L_{e}$ using the following definition.

Definition 3. Let $K$ be a model, and $\varphi$ an LTL formula. Assume that $\Pi$ is an UNSAT core of $\mathrm{BMC}_{k}(\mathrm{~K}, \varphi)$ witnessing $K \models_{k} \varphi$. Then, $p$ is locally irrelevant with respect to $\mathrm{BMC}_{k}(\mathrm{~K}, \varphi)$ and $\Pi$ iff for each time instance $i$, either $p_{i}$ does not appear in $\Pi$ or $p_{i}$ is local to either $C L_{e} \cap \Pi$ or $C L_{K} \cap \Pi$.

In EXAMPLE $1, p$ is locally irrelevant since $p_{1}$ only occurs in the clauses of $U$ taken from $C L_{e}$, while $p_{0}$ only appears in $U$ within $C L_{K}$ clauses. Moreover, the UNSAT core of the original problem can be converted to an UNSAT core of the new theory, thus proving that $p$ is vacuous. Specifically, $U=\left\{\left(p_{0}\right),\left(\neg p_{0}, q_{1}\right),\left(\neg p_{1}\right),\left(p_{1}, \neg q_{1}\right)\right\}$ is the UNSAT core of the original problem, so substituting $x$ for $p$ in the clauses of $U$ that came from $C L_{e}$ gives

$$
U^{\prime}=\left\{\left(p_{0}\right),\left(\neg p_{0}, q_{1}\right),\left(\neg x_{1}\right),\left(x_{1}, \neg q_{1}\right)\right\} .
$$

This is a subset of

$$
\begin{aligned}
& B M C_{1}(K, \varphi[p \leftarrow x])=\left\{\left(p_{0}\right),\left(q_{0}\right),\left(\neg p_{0}, q_{1}\right),\left(\neg x_{1}\right),\right. \\
& \left.\left(x_{1}, \neg q_{1}\right)\right\},
\end{aligned}
$$

so it is a candidate for the new UNSAT core. The substitution may have prevented the resolutions necessary to derive the empty clause. However, Figure 3 shows a proof that $U^{\prime}$ is also unsatisfiable. In this case, it was possible to substitute $x_{i}$ for $p_{i}$ in the clauses coming from $C L_{e}$ in the original UNSAT core and create an UNSAT core for $B M C_{k}(K, \varphi[p \leftarrow x])$. In fact, this observation applies to all cases of local irrelevance by Theorem 2 . Therefore, Definition 3 specifies an algorithm to detect some vacuous variables.
Theorem 2. If $p$ is locally irrelevant with respect to $B_{M} C_{k}(K, \varphi)$ and $\Pi$, then $\varphi$ is $k$-step p-vacuous.

Proof: Let $B M C_{k}(K, \varphi)=C L_{K} \cup C L_{e}$ and $U$ be the UNSAT core returned by the SAT solver for $B M C_{k}(K, \varphi)$. Assume that $p$ is locally irrelevant in $B M C_{k}(K, \varphi)$. So for all $p_{i}$, either $p_{i}$ does not appear in $U$, or $p_{i}$ is local to $C L_{e} \cap U=U_{e}$ or to $C L_{K} \cap U=U_{K}$ by Definition 3. Let $U_{e^{\prime}}$ be $U_{e}$ with each occurence of $p_{i}$ replaced by $x_{i}$. Since each $p_{i}$ that has been replaced is local to $U_{e}$, and $U_{K} \cup U_{e}=U$ is unsatisfiable, then $U_{K} \cup U_{e^{\prime}}$ is also unsatisfiable. Since $U_{e^{\prime}} \subseteq C L_{e}\left[p_{i} \leftarrow x_{i} \mid 0 \leq\right.$ $i<k]$, the set of clauses $C L_{K} \cup C L_{e}\left[p_{i} \leftarrow x_{i} \mid 0 \leq i<k\right]$ is unsatisfiable as well. Therefore, $K \models_{k} \varphi[p \leftarrow x]$ holds, so $\varphi$ is $p$-vacuous.

Unfortunately, if a variable $p$ is not locally irrelevant in an UNSAT core, the formula can still be $p$-vacuous, as shown by the following example.

EXAMPLE 2. Consider a Kripke structure with atomic propositions $r, p$ and $q$ whose initial state is given by the constraint: Init $=\neg r \wedge p \wedge q$. The formula $\varphi=\neg p \vee q$ is $p$-vacuous in the initial state. Let us assume that the zero-step BMC problem is encoded in CNF as follows:

$$
\begin{gathered}
C L_{K}=\left(\neg r_{0}\right)\left(r_{0} \vee p_{0}\right)\left(\neg p_{0} \vee q_{0}\right) \\
C L_{e}=\left(p_{0}\right)\left(\neg p_{0} \vee \neg q_{0}\right)
\end{gathered}
$$

There are several resolution proofs that can establish unsatisfiability of $C L_{K} \cup C L_{e}$; one such proof is shown in Figure 2. In none of the proofs is $p$ locally irrelevant with respect to $C L_{e}$ and $C L_{K}$.

The problem with local irrelevance is that it is impossible to tell if a variable is going to be used in a resolution joining $C L_{K}$ and $C L_{e}$ clauses based on the UNSAT core alone.

\subsection{Peripherality}

In Section 4.1, two vacuity detection methods based on examining the variables in the UNSAT core were found to fall short of completeness. It was seen that even if every possible resolution proof could be analyzed, irrelevance and local irrelevance still might fail to detect existing vacuity. Here, we extend the analysis to the resolution proof's structure. The resulting peripherality algorithm is superior, since it guarantees vacuity will be found if all possible resolution proofs are considered.

The limitations of detecting vacuity based only on the UNSAT core were demonstrated in EXAMPLE 2. By examining the resolution proof in Figure 2, we see that although $p_{0}$ appears both in $C L_{K}$ clauses and in $C L_{e}$ clauses, it is always resolved "locally". That is, if we resolve two clauses $c_{1}=\left(\ldots, p_{i}, \ldots\right)$ and $c_{2}=\left(\ldots, \neg p_{i}, \ldots\right), p_{i}$ and $\neg p_{i}$ must have been preserved from their original source in some set of root clauses. If all the originating root clauses belong to $C L_{K}$ or all belong to $C L_{e}$, then $p_{i}$ is being resolved on locally. In this case, we can replace $p_{i}$ in either set of clauses without affecting their unsatisfiability. For example, in Figure 2, $p_{0}$ can 


$$
\begin{aligned}
& L(c): \text { clause } c \text {, variable } p \rightarrow\left\{{ }^{\prime} \emptyset \text { ' , 'A', 'B', 'AB' }\right\} \\
& \text { - if } c \in \operatorname{Roots}(\Pi) \text { then } \\
& L(c)= \begin{cases}\text { ' } ’ & \text { if } p \notin c \\
\text { 'A' } & \text { if } p \in c \wedge c \in A \\
\text { 'B' } & \text { if } p \in c \wedge c \in B\end{cases}
\end{aligned}
$$

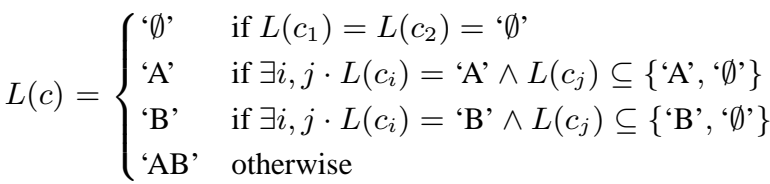

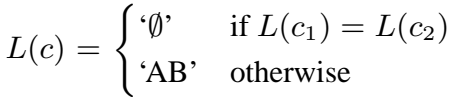

Fig. 4: Labeling function for the peripherality algorithm.

$$
S(l, c)= \begin{cases}\emptyset & \text { if } l \notin c \\ c & \text { if } c \in \operatorname{Roots}(\Pi) \wedge l \in c \\ S\left(l, c_{1}\right) \cup S\left(l, c_{2}\right) & \text { if } c_{1} \text { and } c_{2} \text { are parents } \\ & \text { of } c \wedge l \in c\end{cases}
$$

Fig. 5: Definition of $S(l, c)$.

be replaced in $C L_{e}$ by a new unconstrained variable $x_{0}$. This intuition is formalized below.

Given a resolution proof $\Pi$, a variable $l$, and a clause $c$, we denote by $S(l, c)$ the set of all root clauses that have contributed the variable $l$ to $c . S(l, c)$ is defined recursively as shown in Figure 5. A root clause $c_{r}$ is an element of $S(l, c)$ if it contains a variable $l$ and there exists a path from $c_{r}$ to $c$ that does not contain a resolution on $l$. We can now define peripherality of variables, which captures the conditions when a global variable may not be central to the reason why $\varphi$ holds on $K$.

Definition 4. Let $A$ and $B$ be disjoint sets of clauses such that $C=A \cup B$ is unsatisfiable, and $\Pi$ be a resolution proof establishing unsatisfiability of $C$. Then a variable $l$ is peripheral with respect to $A$ and $B$ iff for every resolution on $l$ between clauses $c_{1}$ and $c_{2}$,

$$
S\left(l, c_{1}\right) \cup S\left(l, c_{2}\right) \subseteq A
$$

or

$$
S\left(l, c_{1}\right) \cup S\left(l, c_{2}\right) \subseteq B .
$$

Within the BMC setting, we have the following definition:

Definition 5. Let $K$ be a model, $\varphi$ be an LTL formula, $\mathrm{BMC}_{k}(\mathrm{~K}, \varphi)$ be a CNF encoding of a BMC problem for $K \models_{k} \varphi$, and $\Pi$ be a proof of unsatisfiability of
$\mathrm{BMC}_{k}(\mathrm{~K}, \varphi) \cdot p$ is peripheral in $\varphi$ iff for each time instance $i, p_{i}$ is peripheral in $\Pi$ with respect to $C L_{e}$ and $C L_{K}$.

If a variable is peripheral, it is vacuous by Theorem 3 .

Theorem 3. Let $\Pi$ be a proof of unsatisfiability of $B M C_{k}(K, \varphi)$. If a variable $p$ of $\varphi$ is peripheral in $\Pi$, then $\varphi$ is $k$-step p-vacuous.

Proof: Let $B M C_{k}(K, \varphi)=C L_{K} \cup C L_{e}$ and $U$ be the UNSAT core returned by the SAT solver for $B M C_{k}(K, \varphi)$. Assume that $p$ is peripheral in $B M C_{k}(K, \varphi)$. Let $U_{e^{\prime}}$ be the result of replacing each $p_{i}$ with $x_{i}$ in $C L_{e} \cap U$. Then $\left(C L_{K} \cap U\right) \cup U_{e^{\prime}}$ is still unsatisfiable, since every resolution on $x_{i}$ must be local to $C L_{e} \cap U$, and every resolution on $p_{i}$ must be local to $C L_{K} \cap U$ by the peripherality of $p_{i}$. Since $U_{e^{\prime}} \subseteq C L_{e}\left[p_{i} \leftarrow x_{i} \mid 0 \leq i<\right.$ $k], C L_{K} \cup C L_{e}\left[p_{i} \leftarrow x_{i} \mid 0 \leq i<k\right]$ is unsatisfiable as well. Therefore, $K \models_{k} \varphi[p \leftarrow x]$, and $\varphi$ is $p$-vacuous.

In Figure 2, although $p$ is not locally irrelevant in $\varphi$, it is peripheral, and therefore $\varphi$ is $p$-vacuous. This also demonstrates that peripherality is a strictly stronger notion than local irrelevance. Theorem 4 shows that under our constraints this is the strongest result that we can hope to establish.

Theorem 4. Assume $\varphi$ is $k$-step p-vacuous in $K$. Then, there exists a resolution proof $\Pi$ of unsatisfiability of $\mathrm{BMC}_{k}(\mathrm{~K}, \varphi)$ such that $p$ is peripheral in $\Pi$.

Proof: Assume that $\varphi$ is $p$-vacuous. Then, the BMC problem $B M C_{k}(K, \varphi[p \leftarrow x])=C L_{K} \cup C L_{e}\left[p_{i} \leftarrow x_{i} \mid 0 \leq i<k\right]$ is unsatisfiable, and there exists a resolution proof $\Pi$ establishing this. We show that such a proof can be transformed to a proof of unsatisfiability of $B M C_{k}(K, \varphi)=C L_{K} \cup C L_{e}$ in which each $p_{i}$ is peripheral with respect to $C L_{K}$ and $C L_{e}$.

Let $\Pi$ be a resolution proof of $B M C_{k}(K, \varphi[p \leftarrow x])$. We transform $\Pi$ into a resolution proof $\Pi^{\prime}$ such that (a) $\operatorname{Roots}\left(\Pi^{\prime}\right) \subseteq C L_{K} \cup C L_{e}\left[p_{i} \leftarrow x_{i} \mid 0 \leq i<k\right]$, and (b) $\Pi^{\prime}$ has no clauses that contain both $p_{i}$ and $x_{i}$ for some $0 \leq i<k$.

Let $U_{K}=\operatorname{Roots}(\Pi) \cap C L_{K}$, and $C L_{e^{\prime}}=C L_{e}\left[p_{i} \leftarrow x_{i} \mid\right.$ $0 \leq i<k]$. Note that if $p_{i}$ occurs in any clause of $U_{K}$, it is local 
to $U_{K}$. Let $L$ be the set of all local variables of $U_{K}, C=\exists L \cdot U_{K}$ be a formula resulting from existentially eliminating these local variables, and $C N F(C)$ be the CNF encoding of $C$. Clearly, for any $0 \leq i<k, p_{i}$ does not appear in $C$. The set of clauses $C N F(C) \cup C L_{e^{\prime}}$ is unsatisfiable. Thus, there exists a resolution proof $\Pi^{\prime \prime}$ establishing this such that $\operatorname{Roots}\left(\Pi^{\prime \prime}\right) \subseteq C L_{e^{\prime}} \cup$ $C N F(C)$. Since $U_{K} \Rightarrow C$, for each clause $c \in C$ there exists a resolution proof $\Pi_{c}$ such that Leaf $\left(\Pi_{c}\right)=c$ and $\operatorname{Roots}\left(\Pi_{c}\right) \subseteq$ $U_{K}$. By combining the proofs $\left\{\Pi_{c} \mid c \in \operatorname{CNF}(C)\right\}$ and $\Pi^{\prime \prime}$, we obtain a proof $\Pi^{\prime}$ of unsatisfiability of $U_{K} \cup C L_{e^{\prime}}$ that does not contain a clause with variables $x_{i}$ and $p_{i}$.

Let $\Pi^{\prime \prime \prime}$ be a proof obtained from $\Pi^{\prime}$ by replacing each $x_{i}$ with $p_{i}$, for $0 \leq i<k$. Then, $\Pi^{\prime \prime \prime}$ is a proof of unsatisfiability of $B M C_{k}(K, \varphi)$ in which all $p_{i}$ are peripheral.

This is one of the main contributions of this article: if a variable appears in all proofs, but is detected as peripheral in at least one of these proofs, it is vacuous. Conversely, if a variable appears in all proofs but is not peripheral in any of them, it is definitively not vacuous.

Peripherality of a variable can be detected by traversing the resolution proof from the roots to the leaf, keeping track of the source of the variable in each clause. If $\Pi$ is a resolution proof whose root clauses are divided into two disjoint sets, $A \cup B$, then the labeling function $L$ is defined recursively as shown in Figure 4, where $c$ is used to represent a clause. This labeling function defines an algorithm for detecting peripherality.

A CNF variable $v$ is peripheral iff the label of the empty clause is not 'AB'. Thus, to detect whether a formula $\varphi$ is $p$-vacuous, we need to check that all CNF variables $p_{i}$ corresponding to $p$ (see Section 2) are peripheral. This can be done by applying the labeling function described in Figure 4 with $A=C L_{K}$, and $B=C L_{e}$ for each $p_{i}$.

By applying this labeling function to the proof shown in Figure 2, we can determine that variable $p$ from EXAMPLE 2 is peripheral. Since there is only one time step, $p$ is peripheral iff $p_{0}$ is peripheral.

- First, we name the root clauses, for later reference:

$$
\begin{aligned}
& c_{1}=\left(\neg r_{0}\right) \\
& p_{0} \notin c_{1} \\
& c_{2}=\left(r_{0} \vee p_{0}\right) \\
& p_{0} \in c_{2} \\
& c_{3}=\left(\neg p_{0} \vee q_{0}\right) \\
& p_{0} \in c_{3} \\
& c_{4}=\left(\neg p_{0} \vee \neg q_{0}\right) \\
& p_{0} \in c_{4} \\
& c_{5}=\left(p_{0}\right) \\
& p_{0} \in c_{5} \\
& c_{1} \in A \\
& c_{2} \in A \\
& c_{3} \in A \\
& c_{4} \in B \\
& c_{5} \in B
\end{aligned}
$$

- Second, we identify the derived clauses:

$$
\begin{array}{ll}
c_{6}=c_{1} \wedge c_{2}, v=r_{0} & c_{8}=c_{7} \wedge c_{4}, v=q_{0} \\
c_{7}=c_{6} \wedge c_{3}, v=p_{0} & c_{9}=c_{8} \wedge c_{5}, v=p_{0}
\end{array}
$$

- Third, we apply the labeling function, as shown in Figure 4:

$$
\begin{aligned}
& L\left(c_{1}\right)={ }^{\prime} \emptyset \text { ' } \quad L\left(c_{6}\right)={ }^{\prime} \mathrm{A} ' v \neq p_{0} \\
& L\left(c_{2}\right)={ }^{\prime} \mathrm{A}, \quad L\left(c_{7}\right)={ }^{\prime} \emptyset ' v=p_{0} \\
& L\left(c_{3}\right)={ }^{\circ} \mathrm{A} \text { ' } L\left(c_{8}\right)={ }^{\prime} \mathrm{B} ' v \neq p_{0} \\
& L\left(c_{4}\right)={ }^{\circ} \mathrm{B} \text { ' } L\left(c_{9}\right)={ }^{\prime} \emptyset{ }^{\prime} v=p_{0} \\
& L\left(c_{5}\right)={ }^{\prime} \mathrm{B} \text { ' }
\end{aligned}
$$

As $L\left(c_{9}\right) \neq$ 'AB', $p_{0}$ is peripheral, so $p$ is peripheral.

It is also possible to simultaneously keep track of the labels for all CNF variables so that only a single pass through $\Pi$ is needed. The time complexity of the peripherality algorithm is linear in the size of the resolution proof.

Theorem 5. For a resolution proof $\Pi$ that $B M C_{k}(K, \varphi)$ is unsatisfiable, determining which variables of $\varphi$ are peripheral can be done in time linear in the size of $\Pi$.

Proof: To determine which variables of $\varphi$ are peripheral, the labeling algorithm defined in Figure 4 must be run once on $\Pi$ for each variable $p$ in $\varphi$. The labeling algorithm passes through the resolution proof, visiting each node once in breadth-first order. A constant amount of work must be done at each node in order to determine the current node's label based on the labels of its two parents. Therefore, one run of the labeling algorithm takes $O(m)$ time, where $m$ is the number of nodes in $\Pi$. To run the labeling algorithm on all variables in $\varphi$ requires $O(m \times n)$ time, where $n$ is the number of variables in $\Pi$. In fact, the size of $\Pi$ is $O(m \times n)$, because each of the $m$ nodes can have a clause of length at most $n$.

In this section, we defined three methods of detecting vacuity based on examining the UNSAT core and the resolution proof produced by BMC. Our evaluation of these algorithms w.r.t. precision and execution times can be found in Section 6.

The effectiveness of our algorithms depends on the structure of the resolution proof found by the SAT solver. It might be possible to make them more effective by modifying the SAT solver to guide it to a particular kind of a proof (e.g., by changing the decision order heuristic), or to output multiple proofs (if possible). Both of these are likely to significantly change performance characteristics of a fine-tuned SAT solver. For these reason, we have concentrated on using the original resolution proof as is.

\section{The Tool: VaqTree}

We have implemented the algorithms presented in Section 4 in a vacuity detection tool called VaqTree. To our knowledge, this is the first vacuity detection tool for BMC.

The inputs to VaqTree are a model (encoded using the specification language of NuSMV [5]) and an LTL property. The tool generates the vacuity results for each variable present in the property. Vacuity detection in VaqTree proceeds in two phases: a "partial pass" that applies one of our methods, and a "model-checking pass" that completes the analysis using additional model-checking runs. The component diagram for VaqTree is shown in Fig. 6. The four components interact sequentially:

SMV $\rightarrow$ CNF Translator receives as input a SMV file containing a model and a property, which are translated into two separate CNF files, one containing $C L_{K}$ and the other $C L_{e}$, respectively. This translation is done using NuSMV, where we modified its BMC package so as to get the translation as two files $(\approx 40$ lines added to the bmcBmcNon Inc . c file). 


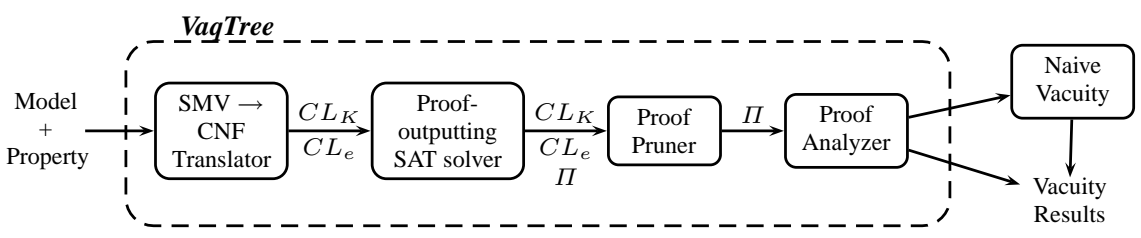

Fig. 6: VaqTree components.

Proof-outputting SAT solver generates the resolution proof $(\Pi)$ for $C L_{K} \cup C L_{e}$. We use MiniSat [10] as it can explicitly generate resolution proofs when checking satisfiability. Instead of using MiniSat's binary proof format, we developed our own XML format which allows easier proof preprocessing and facilitates future incorporation of other SAT solvers.

Proof Pruner receives $C L_{K}, C L_{e}$ and $\Pi$ and applies different preprocessing techniques to $\Pi$, producing a proof from which extraneous chains of resolution have been removed and root clauses are annotated to include their origin $\left(C L_{K}\right.$ or $C L_{e}$ ). This is a new component, written in Java (around 700 lines of code).

Proof Analyzer receives proofs that have been preprocessed using the Proof Pruner component and produces a list of variables found to be vacuous, and a list of variables that need to be tested using the naive method. This is a new component, written in Java (around 1.3k lines of code). The Proof Analyzer outputs the vacuity results for each timed variable $p_{i}$. This information gives an explanation of non-vacuity, indicating which time steps have been important for deciding whether a given variable was vacuous, thus facilitating debugging.

\section{Practical Experience}

We have run VaqTree on two benchmark suites. To evaluate the overall performance of the tool and the effectiveness of our partial vacuity detection methods, we have created a benchmark suite $\mathcal{S}_{\mathcal{A}}$ using various models and properties from the NUSMV distribution. To evaluate the scalability of the tool to industrial models, we have created a benchmark suite $\mathcal{S}_{\mathcal{B}}$ from the models in the IBM Formal Verification Benchmarks Library [14]. These models came with rather simple properties (one temporal operator), and (as expected from an industrial benchmark) did not exhibit a high degree of vacuity. Thus, we used this suite to measure the "worstcase" behavior of the tool, i.e., the amount of overhead incurred by our methods when no vacuity is found.

In the benchmarks, each test case consists of a model $M$, a property $\varphi$, and a bound $k$ such that $M \models_{k} \varphi$. Note that finding an appropriate bound $k$ is orthogonal to $k$-vacuity detection, which explains why our evaluation does not consider the time needed to find $k$. The experiments were performed on a Linux machine with a $2.8 \mathrm{GHz}$ P4 CPU, and $1 \mathrm{~GB}$ of RAM, with up to $700 \mathrm{MB}$ of RAM available to each process.
Currently, VaqTree is limited to proofs with up to 2.5 million resolutions. In $\mathcal{S}_{\mathcal{A}}$, this corresponds to a test case from the asynchronous abp4 model (roughly 30 boolean variables, with $k=19$ ). Our full results are available in Table 1. Below, we discuss results obtained with each benchmark individually.

\subsection{Results obtained with $\mathcal{S}_{\mathcal{A}}$}

This benchmark suite consists of 5 models: abp4, msi_wtrans, pci, and prod-cell from the NUSMV distribution (107 properties) and toyFGS04 from [15] (14 properties). On average, the properties in the suite have 2 temporal operators (from the set $\mathrm{G}, \mathrm{F}, \mathrm{U}$ and $\mathrm{X}$ ), with a maximum of 4 operators, and include both liveness and safety. 99 of the properties exhibit vacuity, and 22 do not.

Scatter plots in Figure 7 compare the execution times of VaqTree (parametrized with irrelevance, local irrelevance, and peripherality), with naive detection for this benchmark. Execution times for naive detection include CNF theory generation and satisfiability testing for each variable of the property. Execution times for VaqTree include the time for the partial pass and the subsequent model-checking pass. Each point in the plot represents a single test case. The $\mathrm{X}$-axis represents the time (in seconds) taken by naive detection. The Y-axis represents the time (in seconds) taken by VaqTree when parameterized by each of our methods. Points below the diagonal indicate where VaqTree was faster than naive detection; points near the diagonal indicate cases where the partial pass found a small percentage of the vacuous variables.

Figure 8 shows that on $\mathcal{S}_{\mathcal{A}}$, VaqTree with irrelevance finds the fewest vacuous variables among our partial methods, as expected from the discussion in Section 4. Although Figure 7(b) and (c) look similar, the numbers (see Table 1) show that local irrelevance is faster than peripherality in $96 \%$ of the cases. This is consistent with the additional work peripherality must perform to analyze the proof tree. A detailed comparison of local irrelevance and naive detection shows that VaqTree with local irrelevance was faster or comparable to naive detection in $95 \%$ of the test cases. VaqTree with local irrelevance was faster than naive detection in $70(58 \%)$ of the test cases, out of which 30 cases were twice as fast, and 20 cases were faster by an order of magnitude. In the remaining 51 cases, local irrelevance was at most $3 \%$ slower in $86 \%$ of these cases. 

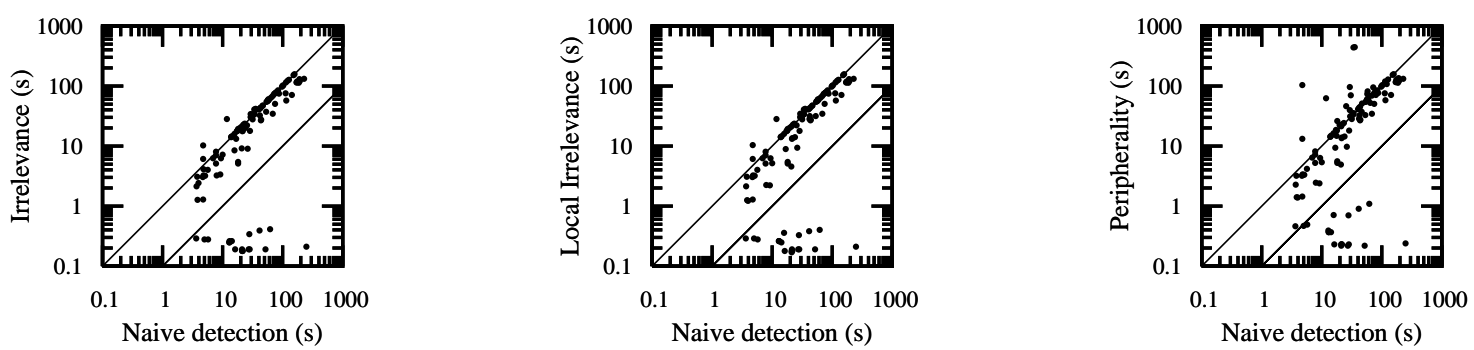

Fig. 7: $\mathcal{S}_{\mathcal{A}}$ : Comparison of execution times. Where applicable, all times include times for both the partial and model-checking passes.
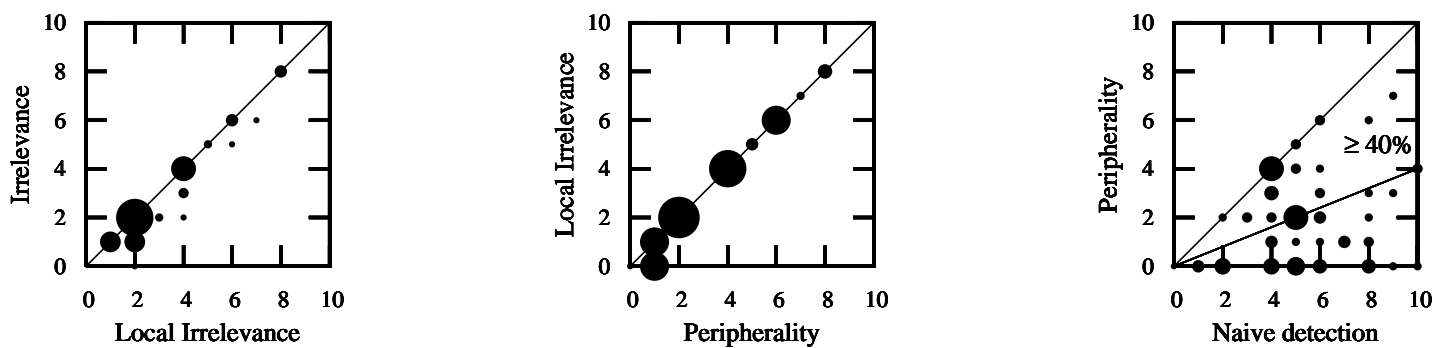

Fig. 8: $\mathcal{S}_{\mathcal{A}}$ : Comparison of the number of vacuous variables detected by partial pass. Larger points represent more test cases than the smaller points.
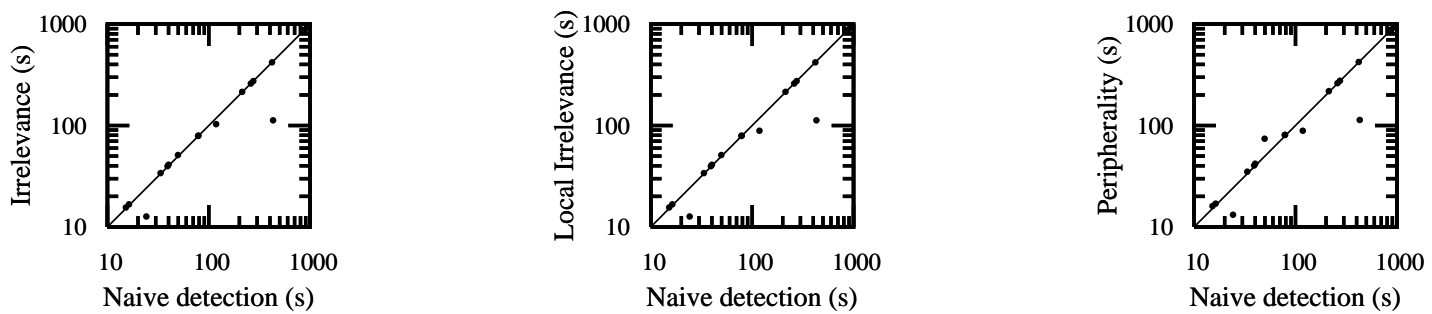

Fig. 9: $\mathcal{S}_{\mathcal{B}}$ : Comparison of execution times. Where applicable, all times include times for both the partial and model-checking passes.

There are 10 cases where VaqTree with peripherality took much longer than naive detection. All of these cases are from the abp4 model, and while they have the largest resolution proofs of the benchmark suite (between 300,000 and 2M clauses), other 300,000-clause test cases did not yield poor performance. We conjecture that the poor performance is due to a low clause/variable ratio [22] which favours naive detection in cases where vacuity is not present. Intuitively, a low ratio indicates that the SAT instance is underconstrained, and so a solution (if it exists) can be found quickly. On the other hand, finding a proof of unsatisfiability in a model with few constraints can be more difficult. Naive detection on a nonvacuous property requires solving satisfiable SAT instances, since replacing variables falsifies the property. However, peripherality on a non-vacuous property requires time linear in the size of the resolution proof obtained from the original model-checking run. If all of these SAT instances have a low clause/variable ratio, naive detection can be much faster than peripherality. This situation was only observed on the abp4 model, with clause/variable ratio of 1.5-1.8 - signifi- cantly lower than any other test case with large proofs and without vacuity.

We now turn to measuring the effectiveness of our methods, using the number of vacuous variables found during the partial pass as a metric (see the scatter plots in Figure 8). This number indicates how many additional model-checking runs are needed to complete vacuity detection. Since our partial methods can be ordered by increasing precision, Figure 8(a) compares irrelevance and local irrelevance, Figure 8(b) - local irrelevance and peripherality, and Figure 8(c) - peripherality and naive detection. Each point in the plot represents a set of test cases - a larger point means a larger set. The axes show the number of vacuous variables detected by each method. Points below the diagonal indicate where the $\mathrm{X}$-axis method detects more vacuous variables than the Y-axis method. The plots show that local irrelevance is clearly more effective than irrelevance. Contrary to our expectations, peripherality performed exactly as local irrelevance in all but 5 cases. Thus, local irrelevance appears to be more cost-effective. Figure 8(c) shows that our techniques are effective when compared with naive detection: peripherality reduced the number of extra 
model-checking runs by $40 \%$ in 54 out of 99 cases that exhibited vacuity.

\subsection{Results obtained with $\mathcal{S}_{\mathcal{B}}$}

This benchmark suite consists of 13 models from the IBM Formal Verification Benchmarks Library [26] (18 properties). The properties have a single temporal operator ( $G$ or $F)$, and include both safety and liveness. 12 of the properties exhibit vacuity, and 6 do not. To evaluate the scalability of VaqTree to industrial models, we must first determine a reasonable bound such that $M \models_{k} \varphi$. For this benchmark, we picked depth $k=20$, which is in line with the bounds used for analyzing these models in [26, Sec. 2]. At this $k$, some of the models where too large to analyze using VaqTree, and some of the properties did not hold. This is why we only report data for 13 models from this benchmark.

Table 1 shows detailed results of our experiments. In this table, column "Benchmark" indicates the benchmark the test case belongs to; "Test case" is the case's unique identifier inside the benchmark, "Model" is the SMV model tested; "\# var. in $M$ " is the number of variables in the model; " $k$ " is the number of steps used to run BMC; "op. in $\varphi$ " shows the property operators (e.g., $2 G$ means that two $\mathrm{G}$ operators appear in the property); "\# var. in $\varphi$ " is the number of atomic variables present in the property; "\# vac. vars." is the number of vacuous variables; and "\# resol. in $\Pi$ " is the number of resolutions in the resolution proof. The next three columns report the time needed for model-checking: "Gen. CNF" is the time NuSMV took to generate the corresponding CNF theory; "Test SAT" and "Gen. $\Pi$ " are the time MiniSat took to test satisfiability and generate the corresponding resolution proof respectively; and "Total" is the sum of the previous three columns.

For the naive method, we report the total times for the CNF theory generation ("Gen. CNF") and for satisfiability testing ("Test SAT"). One CNF theory is produced per atomic variable. For irrelevance, local irrelevance and peripherality, we report how many vacuous variables were found by the partial pass ("\# vac. vars. found"), how long VaqTree took to do the corresponding analysis ("Anal.") and how much time was needed to do the completing pass ("Extra runs").

For example, test case $\mathcal{S}_{\mathcal{B}} \mathbf{1 6}$ analyzes an eight-variable, one temporal operator $(\mathrm{G})$ property of the IBM_FV_2002_23 model (which has 103 variables). All eight of these variables are vacuous. The resolution proof generated when $k=20$ has 7,618 resolutions. This property was checked in $11.29 \mathrm{sec}-$ onds. Naive vacuity detection required eight model-checking runs, taking 115.68 seconds to generate the corresponding CNF theories and 2.36 seconds to test their satisfiability, requiring a total of 118.04 seconds. Irrelevance took $0.36 \mathrm{sec}-$ onds to find one of the vacuous variables during the partial pass. It then took 103.01 seconds to carry out the completing pass, so the total time required by irrelevance to find all eight vacuous variables is 103.37 seconds. Local irrelevance took 0.35 seconds to analyze the resolution proof, finding the same vacuous variable as irrelevance, plus an additional vacuous variable. It takes 88.41 seconds to run the completing pass, so the total time required by local irrelevance is 88.76 seconds. Finally, peripherality took 0.74 seconds to execute the partial pass and found the same two vacuous variables; it also required 88.41 seconds to run the completing pass, taking a total of 89.15 seconds to produce complete results for test case $\mathcal{S}_{\mathcal{B}} \mathbf{1 6}$.

Our results clearly show that proof sizes for the $\mathcal{S}_{\mathcal{B}}$ benchmark can be handled by VaqTree. Interestingly, these are in the same range as proof sizes for $\mathcal{S}_{\mathcal{A}}$. This could be explained by the fact that even though these models are more complex, the properties are simpler.

Scatter plots in Figure 9 compare the execution times of VaqTree parametrized with local irrelevance and peripherality, with naive detection for this benchmark. Execution times are measured as described in Section 6.1, and the graphs are interpreted in the same way as those in Figure 7. Since $\mathcal{S}_{\mathcal{B}}$ had low vacuity, we did not expect our techniques to find it without the help of naive detection. However, graphs in Figure 9 show that our techniques do in fact detect vacuity, as indicated by the points that appear below the diagonal. Both local irrelevance and peripherality detect the same amount of vacuity in $\mathcal{S}_{\mathcal{B}}$, but local irrelevance is slightly faster than peripherality.

Surprisingly, peripherality introduces a low overhead in this benchmark - points over the diagonal are near it, unlike what we see in Figure 7. To explain this behavior, we hypothesized that in non-vacuous cases with low clause/variable ratios and large proofs, peripherality is much slower than naive detection. In $\mathcal{S}_{\mathcal{B}}$, we found that 15 of the test cases have a clause/variable ratio between 2.62-3.66, much higher than the ratios encountered in $\mathcal{S}_{\mathcal{A}}$. The remaining three cases had ratios in the same range as the abp04 model. However, two of these produce trivial proofs, and the last one exhibits vacuity. These results empirically support our hypothesis.

\subsection{Conclusions}

In summary, we observed that local irrelevance performs best out of our proposed partial methods, finding most vacuity in the least amount of time. In $95 \%$ of both benchmark suites, we found VaqTree with local irrelevance to be at most 3\% slower, and usually much faster, than the naive detection. In several tests of the $\mathcal{S}_{\mathcal{A}}$ benchmark, peripherality was noticeably slower than naive detection. On the industrial benchmark $\mathcal{S}_{\mathcal{B}}$, the overhead produced by peripherality was negligible. Interestingly, this suggests that peripherality may be a viable alternative to local irrelevance on industrial models. We plan to investigate this further in the future. Thus, we believe that both local irrelevance and peripherality can be used to speed up naive detection. We plan to enhance our methods by developing a heuristic based on the clause/variable ratio and proof size that indicates when naive detection should be applied instead. 


\section{Summary and Related Work}

In this article, we showed how to exploit the UNSAT core and resolution proof produced by a successful run of BMC for vacuity detection. We introduced three vacuity detection methods that can be applied with little overhead after one model-checking run in order to quickly identify vacuous variables and reduce the number of additional model-checking runs required. Two of these methods, irrelevance and local irrelevance, exploit the UNSAT core, and the third, peripherality, is based on analyzing the resolution proof. We built a tool VaqTree, which is based on these methods, and showed that it is effective for speeding up vacuity detection.

Related work on vacuity detection has been described in Section 3. Additionally, our work is related to research in declarative modeling. In particular, our use of the UNSAT core to detect vacuity was inspired by [23], which addresses the problem of identifying overconstraint in declarative models. While similar in spirit to vacuity detection in model checking, declarative models have no explicit transition relation; instead, transitions are expressed with constraints [17,25]. An overconstraint occurs when the model satisfies a safety property because all violations of the formula have been accidentally ruled out by the declared constraints. In order to detect such overconstraints, [23] introduces the idea of core extraction: declarative models are reduced to SAT instances, from which an UNSAT core can be extracted if the property holds. If a constraint's clauses do not appear in the UNSAT core, the constraint is called irrelevant, and is a source of overconstraint (similar to Definition 2). The cone-of-influence technique [7] is also similar to Definition 2. However, as both of these techniques are model-based, neither can be used to detect vacuity.

Our experiments show that local irrelevance and peripherality can detect more vacuous variables than irrelevance. Therefore, detecting overconstraint in declarative models may also benefit from methods that analyze the structure of the resolution proof. In the future, we propose to investigate how a notion equivalent to peripherality can be defined in the declarative setting. Another goal of future work is to increase the power of resolution proof-based vacuity detection methods. In this article, we restricted ourselves to using results of only one BMC run, and to methods with linear time complexity in the size of the resolution proof or better. However, it is possible that the most optimal trade-off between speed and effectiveness of vacuity detection algorithms lies in the domain of multiple resolution proofs, where we can find the minimal UNSAT core [11] or reduce the resolution proof using interpolation [8].

McMillan [19] uses interpolation to prove that a particular bound is sufficient to imply the unbounded satisfaction of a BMC problem. We intend to combine our techniques with this algorithm in order to prove that bounded vacuity for the correct $k$ implies that the property also holds vacuously in the unbounded case.

Interpolation can also be used to detect vacuity. Given two sets of clauses, $A$ and $B$, such that $A \cup B$ is unsatis- fiable, an interpolant $C$ is a set of clauses whose variables appear in both $A$ and $B$, such that $B \cup C$ is unsatisfiable and $A \Rightarrow C$ [16]. Intuitively, if $C$ is minimal, then $C$ is the reason why $A \cup B$ is unsatisfiable. This intuition suggests that if an interpolant of $C L_{K}$ and $C L_{e}$ could be found, then all variables not appearing in it could be considered vacuous. However, we did not include this technique in our empirical evaluation, as our interpolant generator was comparatively slower.

Another means of speeding vacuity detection for BMC is to iteratively check the $k$-step vacuity of each variable starting with $k=0$. Since $K \nvdash_{k_{1}} \varphi[p \leftarrow x]$ implies $K \not \nvdash_{k_{2}} \varphi[p \leftarrow x]$ for all $k_{2}>k_{1}$, if a variable is proven non-vacuous at some step $k$, then it can be omitted from subsequent checks of higher $k$. This method is orthogonal to our techniques, and the vacuity detection at each step could be carried out by VaqTree.

Acknowledgements. A preliminary version of this article has appeared in [24]. We are grateful to anonymous referees for helping improve the presentation and technical clarity of this article. This work was supported in part by NSERC, OGS, and IBM.

\section{References}

1. R. Armoni, L. Fix, A. Flaisher, O. Grumberg, N. Piterman, A. Tiemeyer, and M. Vardi. "Enhanced Vacuity Detection in Linear Temporal Logic". In Proceedings of the 15th International Conference on Computer Aided Verification (CAV'03), volume 2725 of Lecture Notes in Computer Science, pages 368380, 2003.

2. I. Beer, S. Ben-David, C. Eisner, and Y. Rodeh. "Efficient Detection of Vacuity in ACTL Formulas". In Proceedings of the 9th International Conference on Computer Aided Verification (CAV'97), volume 1254 of Lecture Notes in Computer Science, pages 279-290, 1997.

3. I. Beer, S. Ben-David, C. Eisner, and Y. Rodeh. "Efficient Detection of Vacuity in Temporal Model Checking". Formal Methods in System Design (FMSD), 18(2):141-163, March 2001.

4. A. Biere, A. Cimatti, E. M. Clarke, and Y. Zhu. "Symbolic Model Checking without BDDs". In Proceedings of the 5th International Conference on Tools and Algorithms for the Construction and Analysis of Systems (TACAS'99), volume 1579 of Lecture Notes in Computer Science, 1999.

5. A. Cimatti, E. M. Clarke, E. Giunchiglia, F. Giunchiglia, M. Pistore, M. Roveri, R. Sebastiani, and A. Tacchella. "NUSMV 2: An OpenSource Tool for Symbolic Model Checking". In Proceedings of the 14th International Conference on Computer Aided Verification (CAV'O2), volume 2404 of Lecture Notes in Computer Science, pages 359-364, 2002.

6. A. Cimatti, M. Pistore, M. Roveri, and R. Sebastiani. "Improving the Encoding of LTL Model Checking into SAT". In Proceedings of the 3rd International Workshop on Verification, Model Checking, and Abstract Interpretation (VMCAI'02), volume 2294 of Lecture Notes in Computer Science, pages 196207, 2002.

7. E. Clarke, O. Grumberg, and D. Peled. Model Checking. MIT Press, 1999.

8. W. Craig. "Linear Reasoning. A New Form of the HerbrandGentzen Theorem". Journal of Symbolic Logic (JSL), 22(3):250-268, 1957. 
9. Y. Dong, B. Sarna-Starosta, C.R. Ramakrishnan, and S. A. Smolka. "Vacuity Checking in the Modal Mu-Calculus". In Proceedings of the 9th International Conference on Algebraic Methodology and Software Technology (AMAST'02), volume 2422 of Lecture Notes in Computer Science, pages 147-162. Springer-Verlag, 2002.

10. N. Een and N. Sörensson. The MiniSAT Page. http://www.cs.chalmers.se/Cs/Research/ FormalMethods/MiniSat/Main.html, April 2006.

11. R. Gershman, M. Koifman, and O. Strichman. "Deriving Small Unsatisfiable Cores with Dominators". In Proceedings of the 18th International Conference on Computer Aided Verification (CAV'06), volume 4144 of Lecture Notes in Computer Science, pages 109-122, 2006.

12. A. Gurfinkel and M. Chechik. "Extending Extended Vacuity". In Proceedings of the 5th International Conference on Formal Methods in Computer-Aided Design (FMCAD'04), volume 3312 of Lecture Notes in Computer Science, pages 306321, 2004.

13. A. Gurfinkel and M. Chechik. "How Vacuous Is Vacuous?". In Proceedings of the 10th International Conference on Tools and Algorithms for the Construction and Analysis of Systems (TACAS'04), volume 2988 of Lecture Notes in Computer Science, pages 451-466, 2004.

14. IBM Haifa. CNF Benchmarks from IBM Formal Verification Benchmarks Library, 2007.

15. M. Heimdahl, S. Rayadurgam, W. Visser, G. Devaraj, and J. Gao. "Auto-generating Test Sequences Using Model Checkers: A Case Study". In Proceedings of the Third International Workshop on Formal Approaches to Testing of Software (FATES'03), volume 2931 of Lecture Notes in Computer Science, pages 42-59, 2003.

16. T. Henzinger, R. Jhala, R. Majumdar, and K. McMillan. "Abstractions from Proofs". In Proceedings of the 31st ACM SIGPLAN-SIGACT Symposium on Principles of Programming Langauges (POPL'04), pages 232-244, 2004.

17. D. Jackson. AlLOY: a Lightweight Object Modelling Notation. ACM Transactions on Software Engineering and Methodology (TOSEM), 11(2):256-290, April 2002.

18. O. Kupferman and M. Vardi. "Vacuity Detection in Temporal Model Checking". In Proceedings of the 8th Advanced Research Working Conference on Correct Hardware Design and Verification Methods (CHARME'99), volume 1703 of Lecture Notes in Computer Science, pages 82-96, 1999.

19. K. McMillan. "Interpolation and SAT-Based Model Checking”. In Proceedings of the 15th International Conference on Computer Aided Verification (CAV'03), volume 2725 of Lecture Notes in Computer Science, pages 1-13, 2003.

20. K. Namjoshi. "An Efficiently Checkable, Proof-Based Formulation of Vacuity in Model Checking". In Proceedings of the 16th International Conference on Computer Aided Verification (CAV'04), volume 3114 of Lecture Notes in Computer Science, pages 57-69, 2004.

21. M. Purandare and F. Somenzi. "Vacuum Cleaning CTL Formulae". In Proceedings of the 14th International Conference on Computer Aided Verification (CAV'02), volume 2404 of Lecture Notes in Computer Science, pages 485-499, 2002.

22. B. Selman, D. Mitchell, and H. Levesque. "Generating Hard Satisfiability Problems". Artificial Intelligence, 81(1-2):17-29, March 1996.

23. I. Shlyakhter, R. Seater, D. Jackson, M. Sridharan, and M. Taghdiri. "Debugging Overconstrained Declarative Models Using Unsatisfiable Cores". In Proceedings of the 18th
International Conference on Automated Software Engineering (ASE'03), pages 94-105, October 2003.

24. J. Simmonds, J. Davies, A. Gurinkel, and M. Chechik. "Exploiting Resolution Proofs to Speed Up LTL Vacuity Detection for BMC". In Proceedings of the 7th International Conference on Formal Methods in Computer-Aided Design (FMCAD'07), 2007.

25. J. M. Spivey. The Z Notation: a Reference Manual. Prentice Hall, 1992.

26. Emmanuel Zarpas. "Benchmarking SAT Solvers for Bounded Model Checking". In Proceedings of the 8th International Symposium on the Theory and Applications of Satisfiability Testing (SAT'05), volume 3569 of Lecture Notes in Computer Science, pages 340-354, 2005.

27. L. Zhang and Z. Fu. Boolean Satisfiability Research Group at Princeton. http: //www.princeton.edu/ Chaff/, September 2006.

28. L. Zhang and S. Malik. "Validating SAT Solvers Using an Independent Resolution-Based Checker: Practical Implementations and Other Applications". In Proceedings of the International Conference and Exposition on Design, Automation and Test in Europe (DATE'03), pages 10880-10885, 2003. 
Table 1: Statistics for vacuity detection experiments on NuSMV distribution and other examples.

\begin{tabular}{|c|c|c|c|c|c|c|c|c|c|c|c|c|c|c|c|c|c|c|c|c|c|c|c|c|c|c|c|}
\hline \multirow{2}{*}{\multicolumn{2}{|c|}{$\begin{array}{c}\text { Bench-Tes } \\
\text { mark cas }\end{array}$}} & \multirow[t]{2}{*}{ Model $(M)$} & \multirow{2}{*}{$\left.$\begin{tabular}{|l|}
$=\mathbf{v a r} . \mathbf{k}$ \\
in $M$
\end{tabular}\right|$^{-}$} & \multirow{2}{*}{\multicolumn{2}{|c|}{\begin{tabular}{|l|l|} 
op. & F \\
in $\varphi$ & i
\end{tabular}}} & \multirow{2}{*}{$\begin{array}{l}\# \text { var.\# } \\
\text { in } \varphi\end{array}$} & \multirow{2}{*}{$\begin{array}{l}7 \text { vac. } \\
\text { vars. }\end{array}$} & \multirow{2}{*}{\begin{tabular}{|r|}
$\#$ resol. \\
in $\Pi$
\end{tabular}} & & del Che & ecking & & & Naive & & & Irrelev & vance & & Loca & IIrrele & evance (I & & & eripher: & ality (P) & \\
\hline & & & & & & & & & \begin{tabular}{|r|} 
Gen. \\
$\mathrm{CNF}(\mathrm{s})$
\end{tabular} & Test & Gen. & Total & Gen. & Test & \begin{tabular}{|l|} 
Total \\
\end{tabular} & $\begin{array}{l}\# \\
\text { vac. } \\
\text { vars. } \\
\text { found }\end{array}$ & Anal. & Extra & Total & $\begin{array}{l}\# \\
\text { vac. } \\
\text { vars. } \\
\text { found }\end{array}$ & $\begin{array}{r}\text { Anal. } \\
\text { (s) }\end{array}$ & $\begin{array}{c}\text { Extra } \\
\text { runs (s) }\end{array}$ & Total & $\begin{array}{l}\# \\
\text { vac. } \\
\text { vars. } \\
\text { found }\end{array}$ & \begin{tabular}{|r|} 
Anal. \\
\\
$(\mathrm{s})$
\end{tabular} & $\begin{array}{c}\text { Extra } \\
\text { runs (s) }\end{array}$ & \begin{tabular}{|l|} 
Total \\
\end{tabular} \\
\hline $\mathcal{S}_{\mathcal{A}}$ & 1 & pci & \begin{tabular}{l|l}
40 & 1
\end{tabular} & \begin{tabular}{l|l}
13 & $\mathrm{G}$, \\
\end{tabular} & & 4 & 1 & 19792 & 4.69 & 0.23 & 5.9 & 10.82 & 20.66 & 2.77 & \begin{tabular}{|l|}
23.43 \\
\end{tabular} & 0 & 0.34 & 23.43 & 23.77 & 0 & 0.34 & 23.43 & 23.77 & 0 & 0.81 & 23.43 & 24.24 \\
\hline $\mathcal{S}_{\mathcal{A}}$ & 3 & pci & $40 \quad 1$ & $13 \mathrm{G}$, & & 4 & 3 & 1649 & 5.09 & 0.13 & 5.32 & 10.54 & 12.03 & 2.14 & 14.17 & 3 & 0.26 & & 0.26 & 3 & 0.25 & 0 & 0.25 & 3 & 0.37 & & 0.37 \\
\hline $\mathcal{S}_{\mathcal{A}}$ & 4 & pci & \begin{tabular}{l|l}
40 & 1 \\
\end{tabular} & $13 \mathrm{G}$, & & 3 & 1 & 7725 & 4.80 & 0.18 & 5.65 & 10.63 & 12.68 & 1.73 & 14.41 & 0 & 0.29 & 14.41 & 14.7 & 0 & 0.29 & 14.41 & 14.70 & 0 & 0.50 & 14.41 & 14.91 \\
\hline $\mathcal{S}_{\mathcal{A}}$ & 5 & pci & $\begin{array}{lll}40 & 1\end{array}$ & $\begin{array}{ll}13 \mathrm{G} \text {, } \\
\text {, }\end{array}$ & & 3 & 1 & 7555 & 4.76 & 0.18 & 5.55 & 10.49 & 12.36 & 1.56 & 13.92 & 0 & 0.28 & 13.92 & 14.20 & 0 & 0.28 & 13.92 & 14.20 & 0 & 0.50 & 13.92 & 14.42 \\
\hline $\mathcal{S}_{\mathcal{A}}$ & 6 & pci & \begin{tabular}{l|l}
40 & 1
\end{tabular} & \begin{tabular}{l|l}
13 & $\mathrm{G}$,
\end{tabular} & & 4 & 3 & 1705 & 4.66 & 0.12 & 5.68 & 10.46 & 11.66 & 1.19 & 12.85 & 3 & 0.25 & 0 & 0.25 & 3 & 0.26 & 0 & 0.26 & 3 & 0.39 & 0 & 0.39 \\
\hline $\mathcal{S}_{\mathcal{A}}$ & 7 & pci & 40 & $13 \mid \mathrm{G}$, & & 4 & 3 & 1705 & 4.67 & 0.14 & 5.42 & 10.23 & 11.68 & 1.40 & 13.08 & 3 & 0.25 & & 0.25 & 3 & 0.26 & & 0.26 & 3 & 0.37 & & 0.37 \\
\hline $\mathcal{S}_{\mathcal{A}}$ & 8 & pci & 40 & $13 \mathrm{G}$, & & 5 & 3 & 4283 & 4.95 & 0.22 & 5.59 & 10.76 & 25.85 & 2.89 & 28.74 & 2 & 0.27 & 17.60 & 17.87 & 2 & 0.28 & 17.60 & 17.88 & 2 & 0.47 & 17.60 & 18.07 \\
\hline $\mathcal{S}_{\mathcal{A}}$ & 10 & pci & 40 & \begin{tabular}{l|l}
13 & $\mathrm{~F}, \mathrm{l}$
\end{tabular} & & 3 & 2 & 163 & 4.78 & 0.13 & $\mid 5.49$ & 10.40 & 15.3 & 0.85 & 16.15 & 2 & 0.19 & & 0.19 & 2 & 0.18 & 0 & 0.18 & 2 & 0.23 & & 0.23 \\
\hline $\mathcal{S}_{\mathcal{A}}$ & 11 & pci & \begin{tabular}{l|l}
40 & 1
\end{tabular} & $13 \mid \mathrm{F}, \mathrm{U}$ & $\mathrm{J}, \mathrm{X}$ & 4 & 3 & 165 & 4.7 & 0.13 & \begin{tabular}{|l|} 
\\
\end{tabular} & 10.23 & 20.78 & 0.79 & 21.57 & 3 & 0.18 & 0 & 0.18 & 3 & 0.18 & 0 & 0.18 & 3 & 0.23 & & 0.23 \\
\hline $\mathcal{S}_{\mathcal{A}}$ & 12 & pci & \begin{tabular}{l|l}
40 & 1
\end{tabular} & $13 \mathrm{~F}$, & & 4 & 3 & 162 & 4.85 & 0.14 & 5.41 & 10.40 & 20.25 & 1.01 & 21.26 & 3 & 0.18 & & 0.18 & 3 & 0.19 & 0 & 0.19 & 3 & 0.23 & & 0.23 \\
\hline $\mathcal{S}_{\mathcal{A}}$ & 13 & pci & \begin{tabular}{l|l}
40 & 1
\end{tabular} & \begin{tabular}{l|l}
13 & $\mathrm{~F}, \mathrm{l}$
\end{tabular} & & 4 & 3 & 160 & 5.04 & 0.13 & \begin{tabular}{|l|} 
\\
\end{tabular} .44 \mid & 10.61 & 20.39 & 0.73 & 21.12 & 3 & 0.19 & & 0.19 & 3 & 0.17 & 0 & 0.17 & 3 & 0.23 & 0 & 0.23 \\
\hline $\mathcal{S}_{\mathcal{A}}$ & 14 & pci & \begin{tabular}{l|l}
40 & 1
\end{tabular} & $13 \mid \mathrm{F}, \mathrm{U}$ & $\mathrm{J}, \mathrm{X}$ & 5 & 4 & 164 & 4.79 & 0.14 & 5.34 & 10.27 & 25.81 & 2.34 & 28.15 & 4 & 0.19 & & 0.19 & 4 & 0.19 & 0 & 0.19 & 4 & 0.23 & 0 & 0.23 \\
\hline $\mathcal{S}_{\mathcal{A}}$ & 15 & $\mathrm{pci}$ & 40 & $13 \mathrm{~F}, \mathrm{U}$ & $\mathrm{J}, \mathrm{X}$ & 5 & 4 & 162 & 4.70 & 0.13 & 5.40 & 10.23 & 25.46 & 1.54 & 27.00 & 4 & 0.19 & & 0.19 & 4 & 0.19 & & 0.19 & 4 & 0.22 & & 0.22 \\
\hline $\mathcal{S}_{\mathcal{A}}$ & 16 & pci & 40 & $13 \mathrm{G}$, & & 26 & 19 & 17742 & 4.96 & 0.88 & \begin{tabular}{|l|} 
\\
\end{tabular} & 12.62 & 137.47 & 35.14 & 172.61 & 8 & 0.41 & 115.16 & 115.57 & 8 & 0.42 & 115.16 & 115.58 & 8 & 1.70 & 115.16 & 116.86 \\
\hline $\mathcal{S}_{\mathcal{A}}$ & 17 & pci & \begin{tabular}{l|l}
40 & 1
\end{tabular} & \begin{tabular}{l|l}
13 & $\mathrm{G}$,
\end{tabular} & & 26 & 19 & 26814 & 4.86 & 1.11 & \begin{tabular}{|l|} 
\\
\end{tabular} .87 & 12.84 & 134.29 & 36.13 & 170.42 & 8 & 0.46 & 114.63 & 115.09 & 8 & 0.47 & 114.631 & 115.10 & 8 & 2.38 & 114.63 & 117.01 \\
\hline $\mathcal{S}_{\mathcal{A}}$ & 18 & pci & \begin{tabular}{l|l}
40 & 1
\end{tabular} & \begin{tabular}{l|l}
13 & $\mathrm{G}$,
\end{tabular} & & 26 & 19 & 66883 & 5.07 & 1.24 & 7.87 & 14.18 & 147.93 & 42.23 & 190.16 & 8 & 0.60 & 130.62 & 131.22 & 8 & 0.60 & 130.621 & 131.22 & 8 & 5.67 & 130.62 & 136.29 \\
\hline $\mathcal{S}_{\mathcal{A}}$ & 19 & $\mathrm{pci}$ & 40 & $13 \mathrm{G}$, & & 26 & 19 & 96762 & 4.87 & 2.72 & 10.48 & 18.07 & 133.67 & 41.05 & 174.72 & 8 & 0.74 & 118.51 & 119.25 & 8 & 0.73 & $\mid 18.51$ & 119.24 & 8 & 7.96 & | 118.51 & 126.47 \\
\hline $\mathcal{S}_{\mathcal{A}}$ & 20 & pci & 40 & $\begin{array}{ll}7 & \mathrm{G} \text {, }\end{array}$ & & 5 & 3 & 1526 & 1.56 & 0.08 & 3.26 & 4.90 & 8.73 & 0.64 & 9.37 & 2 & 0.24 & 5.99 & 6.23 & 2 & 0.24 & \begin{tabular}{|l|} 
\\
\end{tabular} & 6.23 & 2 & 0.36 & \begin{tabular}{|l|}
5.99 \\
\end{tabular} & 6.35 \\
\hline $\mathcal{S}_{\mathcal{A}}$ & 23 & prod-cell & 393 & $30 \mathrm{G}$, & & 4 & 4 & 13946 & 11.06 & 0.61 & \begin{tabular}{|l|} 
\\
\end{tabular} .59 & 18.26 & 40.37 & 0.94 & 41.31 & 4 & 0.39 & 0 & 0.39 & 4 & 0.38 & 0 & 0.38 & 4 & 0.90 & & 0.90 \\
\hline $\mathcal{S}_{\mathcal{A}}$ & 24 & prod-cell & 393 & \begin{tabular}{l|l}
30 & $\mathrm{G}$,
\end{tabular} & & 6 & 6 & 67307 & 10.53 & 0.38 & 5.20 & 16.11 & 77.67 & 1.80 & 79.47 & 0 & 0.65 & 79.47 & 80.12 & 0 & 0.64 & 79.47 & 80.11 & 1 & 4.62 & 66.41 & 71.03 \\
\hline $\mathcal{S}_{\mathcal{A}}$ & 25 & d-cell & 393 & \begin{tabular}{l|l}
30 & $\mathrm{G}$,
\end{tabular} & & 7 & 7 & 24146 & 11.3 & 0.32 & |4.87 & 16.49 & 85.36 & 1.75 & 87.11 & 1 & 0.55 & 74.66 & 75.21 & 1 & 0.54 & 74.66 & 75.20 & 1 & 1.97 & 74.66 & 76.63 \\
\hline $\mathcal{S}_{\mathcal{A}}$ & 26 & prod-cell & 393 & $30 \mathrm{G}$, & & 6 & 6 & 15898 & 9.98 & 0.19 & 4.12 & 14.29 & 75.39 & 1.87 & 77.26 & 0 & 0.44 & 77.26 & 77.70 & 0 & 0.44 & 77.26 & 77.70 & 0 & 1.35 & 77.26 & 78.61 \\
\hline $\mathcal{S}_{\mathcal{A}}$ & 27 & prod-cell & 393 & $30 \mathrm{G}$, & & 5 & 5 & 15734 & 9.50 & 0.28 & \begin{tabular}{|l|} 
\\
\end{tabular} & 14.42 & 62.85 & 1.24 & 64.09 & 0 & 0.42 & 64.09 & 64.51 & 0 & 0.41 & 64.09 & 64.50 & 1 & 1.19 & 51.52 & 52.71 \\
\hline $\mathcal{S}_{\mathcal{A}}$ & 28 & od-cell & 393 & $\begin{array}{ll}30 & \mathrm{G},\end{array}$ & & 4 & 4 & 24615 & 9.25 & 0.16 & 4.18 & 13.59 & 40.61 & 1.29 & 41.90 & 0 & 0.41 & 41.90 & 42.31 & & 0.40 & 41.90 & 42.30 & 0 & 1.47 & 41.90 & 43.37 \\
\hline $\mathcal{S}_{\mathcal{A}}$ & 29 & prod-cell & 393 & \begin{tabular}{l|l}
30 & $\mathrm{G}$,
\end{tabular} & & 8 & 8 & 24747 & 10.34 & 0.23 & \begin{tabular}{|l|} 
\\
\end{tabular} & 15.11 & 100.24 & 1.99 & 102.23 & 0 & 0.52 & 102.23 & 102.75 & 0 & 0.53 & 102.23 & 102.76 & 0 & 2.27 & 102.231 & 104.50 \\
\hline $\mathcal{S}_{\mathcal{A}}$ & 30 & d-cell & 393 & \begin{tabular}{l|l}
30 & $\mathrm{G}$,
\end{tabular} & & 5 & 5 & 15177 & 9.27 & 0.35 & |4.31 & 13.93 & 55.87 & 3.61 & 59.48 & 0 & 0.41 & 59.48 & 59.89 & 0 & 0.40 & 59.48 & 59.88 & 0 & 1.17 & 59.48 & 60.65 \\
\hline $\mathcal{S}_{\mathcal{A}}$ & 31 & prod-cell & 393 & 30 G, & & 4 & 4 & 35955 & 8.89 & 1.01 & \begin{tabular}{|l|} 
\\
\end{tabular} & 16.37 & 43.87 & 2.52 & 46.39 & 0 & 0.51 & 46.39 & 46.90 & 0 & 0.49 & 46.39 & 46.88 & 1 & 1.87 & 35.48 & 37.35 \\
\hline $\mathcal{S}_{\mathcal{A}}$ & 32 & d-cell & 39 & \begin{tabular}{l|l}
30 & $\mathrm{G}$,
\end{tabular} & & 4 & 4 & 101479 & 9.24 & 0.69 & \begin{tabular}{|l|} 
\\
\end{tabular} & 16.15 & 43.35 & 3.18 & 46.53 & 0 & 0.71 & 46.53 & 47.24 & 0 & 0.71 & 46.53 & 47.24 & 0 & 4.63 & 46.53 & 51.16 \\
\hline $\mathcal{S}_{\mathcal{A}}$ & 33 & & 393 & $\begin{array}{ll}30 & \mathrm{G},\end{array}$ & & 6 & 6 & 327 & 9.91 & 0.86 & \begin{tabular}{|l|} 
\\
\end{tabular} & 17.70 & 74.37 & 2.40 & 76.77 & 0 & 0.58 & 76.77 & 77.35 & 0 & 0.57 & 76.77 & 77.34 & 0 & 2.30 & 76.77 & 79.07 \\
\hline $\mathcal{S}_{\mathcal{A}}$ & 34 & prod-cell & 393 & $30 \mathrm{G}$, & & 5 & 5 & 14854 & 9.39 & 0.30 & \begin{tabular}{|l|} 
\\
\end{tabular} & 14.67 & 54.8 & 6.69 & 61.49 & 5 & 0.41 & & 0.41 & 5 & 0.40 & & 0.40 & 5 & 1.09 & & 1.09 \\
\hline $\mathcal{S}_{\mathcal{A}}$ & 35 & & 39 & $30 \mathrm{G}$, & & 5 & 5 & 80108 & 9.10 & 0.99 & 6.81 & 16.90 & 52.65 & 4.40 & 57.05 & 0 & 0.73 & 57.05 & 57.78 & 0 & 0.74 & 57.05 & 57.79 & 0 & 4.36 & 57.05 & 61.41 \\
\hline $\mathcal{S}_{\mathcal{A}}$ & 36 & d-cell & 39 & \begin{tabular}{l|l}
30 & $\mathrm{G}$,
\end{tabular} & & 8 & 8 & 21654 & 11.43 & 0.35 & 4.64 & 16.42 & 118.41 & 2.92 & 121.33 & 0 & 0.52 & 121.33 & 121.85 & 0 & 0.52 & 121.331 & 121.85 & 1 & 1.97 & 106.42 & 108.39 \\
\hline $\mathcal{S}_{\mathcal{A}}$ & 37 & I-cell & 39 & \begin{tabular}{l|l}
30 & $\mathrm{G}$,
\end{tabular} & & 9 & 9 & 59079 & 10.31 & 0.40 & 5.91 & 16.62 & 108.15 & 5.90 & 114.05 & 0 & 0.67 & 114.05 & 114.72 & 0 & 0.66 & $114.05 \mid 1$ & 114.71 & 0 & 5.71 & 114.05 & 119.76 \\
\hline $\mathcal{S}_{\mathcal{A}}$ & 38 & & 39 & $30 \mathrm{G}$, & & 5 & 5 & 27070 & 9.25 & 0.37 & 5.04 & 14.66 & 54.85 & 2.24 & 57.09 & 0 & 0.53 & 57.09 & 57.62 & 0 & 0.54 & \begin{tabular}{|r|}
57.09 \\
\end{tabular} & 57.63 & 0 & 1.72 & $\begin{array}{l}57.09 \\
\end{array}$ & 58.81 \\
\hline $\mathcal{S}_{\mathcal{A}}$ & 39 & prod-cell & 393 & \begin{tabular}{l|l}
30 & $\mathrm{G}$,
\end{tabular} & & 5 & 5 & 32664 & 8.98 & 0.48 & 5.29 & 14.75 & 53.81 & 1.95 & 55.76 & 0 & 0.55 & 55.76 & 56.31 & 0 & 0.55 & 55.76 & 56.31 & 0 & 2.01 & 55.76 & 57.77 \\
\hline $\mathcal{S}_{\mathcal{A}}$ & 40 & & 39 & $\begin{array}{ll}30 & \mathrm{G},\end{array}$ & & 4 & 4 & 393 & 9.18 & 0.44 & 5.15 & 14.77 & 41.74 & 1. & 43.24 & 0 & 0.54 & 43.24 & 43.78 & 0 & 0.54 & 43.24 & 43.78 & 0 & & 43.24 & 45.23 \\
\hline $\mathcal{S}_{\mathcal{A}}$ & 41 & & 39 & $302 \mathrm{G}$, &, $2 \mathrm{~F}$ & 10 & 10 & 20771 & 11.78 & 0.20 & 4.71 & 16.69 & 148.20 & 3.00 & 151.20 & 0 & 0.53 & 151.20 & 151.73 & 0 & 0.53 & 151.201 & 151.73 & 0 & 2.25 & 151.201 & 153.45 \\
\hline $\mathcal{S}_{\mathcal{A}}$ & 42 & prod-cell & 393 & 30 G, & & 8 & 8 & 22039 & 11.42 & 0.72 & \begin{tabular}{|l|} 
\\
\end{tabular} & 18.17 & 123.54 & 3.03 & 126.57 & 0 & 0.54 & 126.57 & 127.11 & 0 & 0.53 & 126.571 & 127.10 & 0 & 1.97 & 126.571 & 128.54 \\
\hline $\mathcal{S}_{\mathcal{A}}$ & 43 & & 39 & $\begin{array}{ll}20 & \mathrm{G},\end{array}$ & & 4 & 4 & 17565 & & 0.11 & 2.81 & 6.92 & 17.37 & 0.35 & 17.72 & 0 & 0.38 & 17.72 & 18. & 0 & 0.38 & $\mid \quad 17.72$ & & 1 & & 13.82 & 14.73 \\
\hline $\mathcal{S}_{\mathcal{A}}$ & 44 & prod-cell & 39 & $\begin{array}{ll}20 & \mathrm{G},\end{array}$ & & 6 & 6 & 23833 & 3.97 & 0.13 & 2.70 & 6.80 & 29.07 & 0.69 & 29.76 & 0 & 0.42 & 29.76 & 30.18 & 0 & 0.42 & 29.76 & 30.18 & 0 & 1.41 & 29.76 & 31.17 \\
\hline $\mathcal{S}_{\mathcal{A}}$ & 45 & $\mathrm{p}$ & 39 & \begin{tabular}{l|l}
20 & $\mathrm{G}$,
\end{tabular} & & 7 & 7 & 11023 & 3.84 & 0.12 & \begin{tabular}{|l|}
2.69 \\
\end{tabular} & 6.65 & 31.11 & 0.66 & 31.77 & 1 & 0.38 & 27.35 & 27.73 & 1 & 0.37 & 27.35 & 27.72 & 1 & 0.90 & 27.35 & 28.25 \\
\hline $\mathcal{S}_{\mathcal{A}}$ & 46 & & 39 & $\begin{array}{ll}20 & \mathrm{G},\end{array}$ & & 6 & 6 & & 3.84 & 0.14 & 3.31 & 7.29 & 25.84 & 0.46 & 26.30 & 4 & 0.36 & 8.63 & 8.99 & 4 & 0.35 & 8.99 & 9.34 & 4 & 0.74 & 8.99 & 9.73 \\
\hline $\mathcal{S}_{\mathcal{A}}$ & 50 & & 392 & $20 \mathrm{G}$, & & 5 & 5 & 59884 & 3.86 & 0.42 & 4.48 & 8.76 & 19.35 & 0.50 & 19.85 & 0 & 0.55 & 19.85 & 20.40 & 0 & 0.55 & 19.85 & 20.40 & 0 & 2.64 & 19.85 & 22.49 \\
\hline $\mathcal{S}_{\mathcal{A}}$ & 51 & $\mathrm{p}$ & 39 & \begin{tabular}{l|l}
20 & $\mathrm{G}$,
\end{tabular} & $\mathrm{F}$ & 4 & 4 & 10793 & 3.84 & 0.18 & 3.23 & 7.25 & 15.07 & 0.66 & 15.73 & 2 & 0.36 & 8.14 & 8.50 & 4 & 0.36 & 0 & 0.36 & 4 & 0.71 & 0 & 0.71 \\
\hline $\mathcal{S}_{\mathcal{A}}$ & 52 & prod-cell & 39 & $20 \mathrm{G}$, & & 4 & 4 & 25764 & 4.01 & 0.16 & 2.84 & 7.01 & 16.81 & 0.52 & 17.33 & 0 & 0.40 & 17.33 & 17.73 & 0 & 0.39 & 17.33 & 17.72 & 0 & 1.15 & 17.33 & 18.48 \\
\hline $\mathcal{S}_{\mathcal{A}}$ & 53 & prod-cell & 39 & $20 \mathrm{G}$, & $\mathrm{F}$ & 6 & 6 & 8120 & 4.15 & 0.15 & 3.33 & 7.63 & 27.20 & 0.52 & 27.72 & 6 & 0.34 & & 0.34 & 6 & 0.33 & 0 & 0.33 & 6 & 0.70 & 0 & 0.70 \\
\hline $\mathcal{S}_{\mathcal{A}}$ & 54 & prod-cell & 39 & \begin{tabular}{l|l}
20 & $\mathrm{G}$,
\end{tabular} & $\mathrm{F}$ & 5 & 5 & 8840 & 3.8 & 0.10 & 2.76 & 6.66 & 19.72 & 1.24 & 20.96 & 3 & 0.33 & 8.80 & 9.13 & 4 & 0.34 & 4.21 & 4.55 & 4 & 0.69 & 4.21 & 4.90 \\
\hline $\mathcal{S}_{\mathcal{A}}$ & 55 & prod-cell & 39 & $\mathrm{G}$, & & 5 & 5 & 25425 & 3.9 & 0.22 & 3.81 & 7.93 & 21.18 & 1.03 & 22.21 & 0 & 0.43 & 22.21 & 22.64 & 0 & 0.42 & 22.21 & 22.63 & 0 & 1.26 & 22.21 & 23.47 \\
\hline
\end{tabular}


Table 1 - Continued

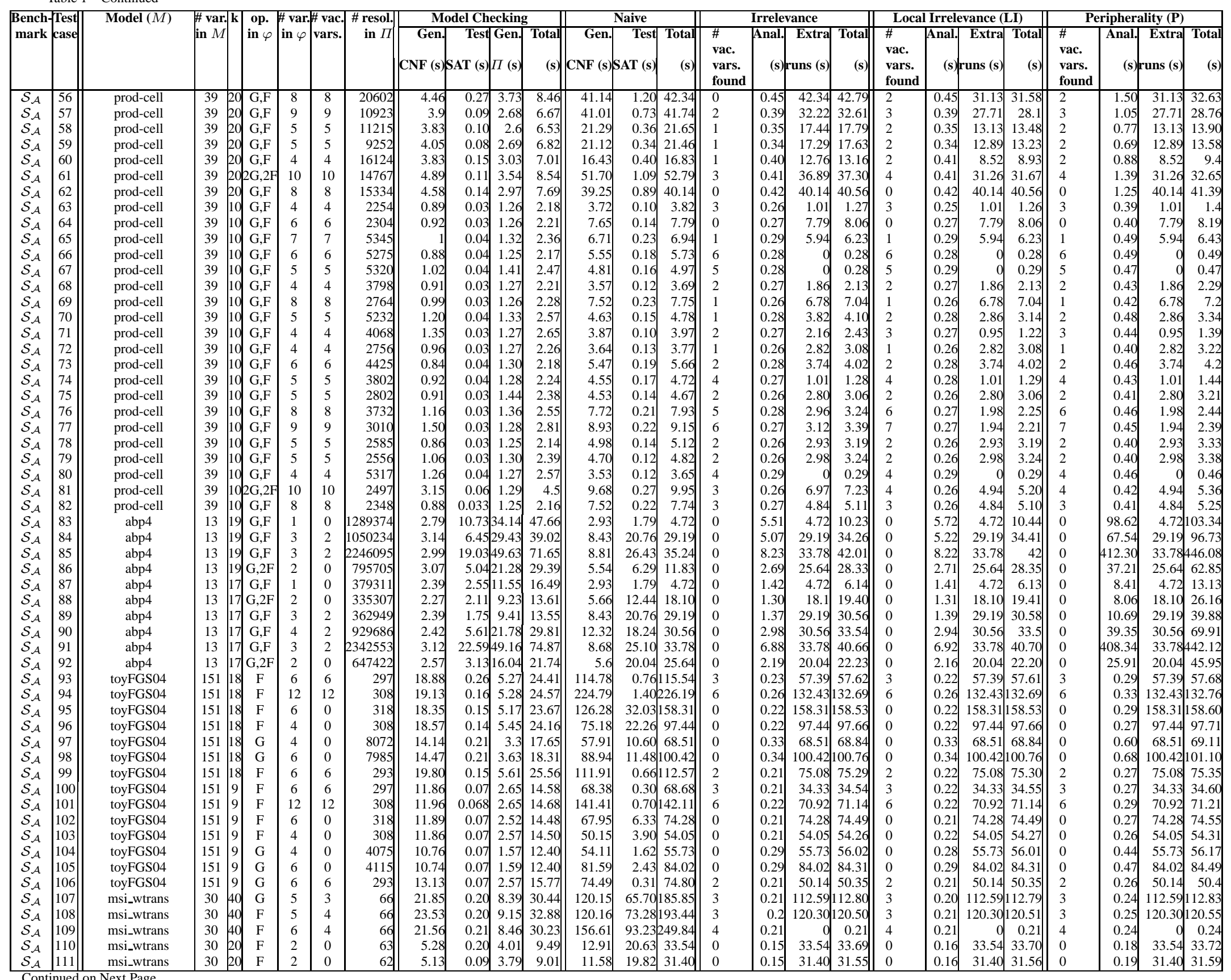


Table 1 - Continued

\begin{tabular}{|c|c|c|c|c|c|c|c|c|c|c|c|c|c|c|c|c|c|c|c|c|c|c|c|c|c|c|}
\hline \multirow{3}{*}{$\begin{array}{l}\text { Bench-T } \\
\text { mark }\end{array}$} & \multirow{3}{*}{ Test } & \multirow[t]{3}{*}{ Model $(M)$} & \multirow{3}{*}{$\begin{array}{l}\# \mathbf{~ v a r} . \mathbf{k} \\
\text { in } M\end{array} \mid$} & \multirow{3}{*}{$\begin{array}{l}\text { op. } \\
\text { in } \varphi\end{array}$} & \multirow{3}{*}{$\mid \begin{array}{l}\# \text { var. } \\
\text { in } \varphi\end{array}$} & \multirow{3}{*}{$\begin{array}{l}\text { \# vac. } \\
\text { vars. }\end{array}$} & \multirow{3}{*}{$\begin{array}{r}\# \text { resol. } \\
\text { in } \Pi\end{array}$} & \multicolumn{4}{|c|}{ Model Checking } & \multicolumn{3}{|c|}{ Naive } & \multicolumn{4}{|c|}{ Irrelevance } & \multicolumn{4}{|c|}{ Local Irrelevance (LI) } & \multicolumn{4}{|c|}{ Peripherality (P) } \\
\hline & & & & & & & & Gen. & Test & Gen. & \begin{tabular}{|l|} 
Total \\
\end{tabular} & Gen. & Test & Total & & Anal. & Extra & \begin{tabular}{|l|} 
Total \\
\end{tabular} & & Anal. & Extra & Total & & Anal. & T. & \\
\hline & & & & & & & & & & $\Pi(\mathrm{s})$ & & $\mathrm{CNF}(\mathrm{s}$ & & (s) & $\begin{array}{l}\text { vac. } \\
\text { vars. } \\
\text { found }\end{array}$ & & runs (s) & (s) & $\begin{array}{l}\text { vac. } \\
\text { vars. } \\
\text { found }\end{array}$ & & runs $(s)$ & (s) & $\begin{array}{l}\text { vac. } \\
\text { vars. } \\
\text { found }\end{array}$ & & runs $(\mathrm{s})$ & \\
\hline $\mathcal{S}_{\mathcal{A}}$ & 112 & msi_wtrans & \begin{tabular}{l|l}
30 & 20 \\
\end{tabular} & $\mathrm{G}$ & 6 & 0 & 374744 & 4.68 & 10.21 & 121.20 & 36.09 & 32.04 & 26.13 & 58.17 & 0 & 1.78 & 58.17 & 59.95 & 0 & 1.80 & 58.17 & 59.97 & 0 & 16.42 & 58.17 & 74.59 \\
\hline $\mathcal{S}_{\mathcal{A}}$ & 113 & msi_wtrans & 3020 & G & 6 & 0 & 274539 & 4.72 & 9.76 & 619.65 & 34.13 & 28.92 & 28.27 & 57.19 & 0 & 1.48 & 57.19 & 58.67 & 0 & 1.51 & 57.19 & 58.70 & 0 & 11.99 & 57.19 & 69.18 \\
\hline $\mathcal{S}_{\mathcal{A}}$ & 114 & msi_wtrans & 3020 & $\mathrm{G}$ & 5 & 3 & 66 & 5.27 & 0.10 & 0 & \begin{tabular}{|l|}
9.22 \\
\end{tabular} & 27.92 & 15.65 & 43.57 & 3 & 0.16 & 26.75 & 26.91 & 3 & 0.16 & 26.75 & 26.91 & 3 & 0.19 & 26.75 & 26.94 \\
\hline $\mathcal{S}_{\mathcal{A}}$ & 115 & msi_wtrans & 3020 & $\mathrm{~F}$ & 5 & 4 & 66 & 5.17 & 0.11 & \begin{tabular}{l|l}
1 & 3.94 \\
$r$
\end{tabular} & 9.22 & 27.5 & 16.62 & 44.12 & 3 & 0.16 & 27.25 & 27.41 & 3 & 0.15 & 27.25 & 27.40 & 3 & 0.19 & 27.25 & 27.44 \\
\hline $\mathcal{S}_{\mathcal{A}}$ & 116 & msi_wtrans & 3020 & $\mathrm{~F}$ & 6 & 4 & 66 & 5.31 & 0.10 & $\begin{array}{l}03.91 \\
0\end{array}$ & 9.32 & 33.3 & 18.60 & 51.90 & 4 & 0.19 & & 0.19 & 4 & 0.19 & 0 & 0.19 & 4 & 0.22 & & 0.22 \\
\hline $\mathcal{S}_{\mathcal{A}}$ & 117 & msi_wtrans & 3020 & $\mathrm{G}$ & 2 & 0 & 1604 & 4.62 & 0.11 & 3.68 & 8.41 & 9.29 & 11.84 & 21.13 & 0 & 0.24 & 21.13 & 21.37 & 0 & 0.24 & 21.13 & 21.37 & 0 & 0.35 & 21.13 & 21.48 \\
\hline $\mathcal{S}_{\mathcal{A}}$ & 118 & msi_wtrans & 3020 & G,F & 1 & 0 & 1001 & 5.41 & 0.10 & $\begin{array}{l}03.94 \\
0\end{array}$ & 9.45 & 5.73 & 8.20 & 13.93 & 0 & 0.22 & 13.93 & 14.15 & 0 & 0.23 & 13.93 & 14.16 & 0 & 0.30 & 13.93 & 14.23 \\
\hline $\mathcal{S}_{\mathcal{A}}$ & 120 & & 3020 & G,F & 2 & 0 & 927 & 5.58 & 0.10 & 0.47 & 6.15 & 12.39 & 18.96 & 31.35 & 0 & 0.22 & 31.35 & 31.57 & 0 & 0.22 & 31.35 & 31.57 & 0 & 0.32 & 31.35 & 31.67 \\
\hline $\mathcal{S}_{\mathcal{A}}$ & 121 & msi_wtrans & 3020 & G,F & 8 & 0 & 371653 & 4.76 & 12.49 & 924.41 & 41.66 & 41.96 & 30.22 & 72.18 & 0 & 1.79 & 72.18 & 73.97 & 0 & 1.80 & 72.18 & 73.98 & 0 & 16.16 & 72.18 & 88.34 \\
\hline $\mathcal{S}_{\mathcal{B}}$ & 1 & IBM_FV_2002_03 & 11120 & $\mathrm{G}$ & 8 & 8 & 7480 & 4.54 & 0.09 & $\begin{array}{l}93.8 \\
9\end{array}$ & 8.43 & 36.21 & 0.67 & 36.88 & 7 & 0.35 & 4.67 & 5.02 & 7 & 0.35 & 4.67 & 5.02 & 7 & 0.74 & 4.67 & 5.41 \\
\hline $\mathcal{S}_{\mathcal{B}}$ & 2 & IBM_FV_2002_04 & 22320 & G & 4 & 3 & 45065 & 7.62 & 0.92 & 5.71 & 14.25 & 29.66 & 3.83 & 33.49 & 0 & 0.59 & 33.49 & 34.08 & 0 & 0.59 & 33.49 & 34.08 & 0 & 1.67 & 33.49 & 35.16 \\
\hline $\mathcal{S}_{\mathcal{B}}$ & 3 & IBM_FV_2002_05 & 31020 & G & 2 & 1 & 32776 & 11.82 & 0.62 & 210.02 & 22.46 & 22.97 & 1.31 & $|24.28|$ & 1 & 0.44 & 12.21 & 12.65 & 1 & 0.44 & 12.21 & 12.65 & 1 & 1.02 & 12.21 & 13.23 \\
\hline $\mathcal{S}_{\mathcal{B}}$ & 4 & IBM_FV_2C & 23320 & $\mathrm{~F}$ & 9 & 9 & & 8.96 & 0.17 & & 9.13 & 81.02 & 1.22 & $82.24 \mid$ & 9 & 0.17 & & 0.17 & 9 & 0.17 & & 0.17 & 9 & 0.17 & & 0.17 \\
\hline $\mathcal{S}_{\mathcal{B}}$ & 5 & IBM_FV_2002_10 & 22420 & G & 3 & 2 & 78523 & 54.23 & 8.45 & 546.09 & $108.77 \mid$ & 165.88 & 93.22 & 259.1 & 0 & 0.7 & 259.1 & 259.8 & 0 & 0.7 & 259.1 & 259.8 & 0 & 2.33 & 259.1 & 261.43 \\
\hline $\mathcal{S}_{\mathcal{B}}$ & 6 & IBM_FV_2002_10 & 22420 & G & 4 & 3 & 177536 & 53.3 & 30.21 & 156.61 & 140.12 & 219.74 & 199.25 & 418.99 & 0 & 1.12 & 418.99 & 420.11 & & 1.12 & 418.99 & 420.11 & 0 & 5.8 & 418.99 & 424.79 \\
\hline $\mathcal{S}_{\mathcal{B}}$ & 7 & IBM_FV_2002_10 & 22420 & G & 4 & 4 & & 53.97 & & 740.84 & 95.78 & 218.45 & 211.21 & 429.66 & 3 & 0.32 & 112.58 & 112.9 & 3 & 0.32 & 112.58 & 112.9 & 3 & 0.61 & 112.58 & 113.19 \\
\hline $\mathcal{S}_{\mathcal{B}}$ & 8 & IBM_FV_2002_10 & 22420 & G & 2 & 0 & 155775 & 54.99 & 9.22 & 246.75 & 110.96 & 108.76 & 165.1 & 273.86 & 0 & 0.99 & 273.86 & 274.85 & & 0.99 & 273.86 & 274.85 & 0 & 3.7 & 273.86 & 277.56 \\
\hline $\mathcal{S}_{\mathcal{B}}$ & 9 & IBM_FV_2002_10 & 22420 & G & 2 & 1 & 19 & 54.96 & 65.43 & 379.32 & 199.71 & 110.2 & 103.82 & 214.02 & 0 & 1. & 214.02 & 215.11 & & & 214.02 & 215.14 & 0 & & 214.02 & 218.52 \\
\hline $\mathcal{S}_{\mathcal{B}}$ & 10 & IBM_FV & 158420 & G & 2 & 0 & 960 & 38.58 & 1.15 & 514.23 & 53.96 & 75.78 & 2.35 & 78.13 & 0 & 1.07 & 78.13 & 79.2 & & 1.08 & 78.13 & 79.21 & 0 & 2.47 & 78.13 & 80.6 \\
\hline $\mathcal{S}_{\mathcal{B}}$ & 13 & IBM_FV_2002_19 & 12120 & G & 1 & 0 & 35769 & 9.56 & 6.49 & 913.31 & 29.36 & 9.65 & 5.52 & 15.17 & 0 & 0.39 & 15.17 & 15.56 & 0 & 0.4 & 15.17 & 15.57 & 0 & 0.83 & 15.17 & 16 \\
\hline $\mathcal{S}_{\mathcal{B}}$ & 14 & IBM_FV_2 & & G & 1 & 0 & 25508 & 8.8 & 5.61 & 11.98 & 26.39 & 8.68 & 7.61 & $16.29 \mid$ & 0 & & 16.29 & 16.6 & 0 & 0.37 & 16.29 & 16.66 & 0 & 0.71 & 16.29 & 17 \\
\hline $\mathcal{S}_{\mathcal{B}}$ & 15 & IBM_FV_2002_22 & 10420 & G & 1 & 0 & 53300 & 14.58 & 7.74 & 417.84 & 40.16 & 14.78 & 24.42 & \begin{tabular}{|r|}
39.2 \\
\end{tabular} & 0 & 0.53 & 39.2 & 39.73 & 0 & 0.53 & 39.2 & 39.73 & 0 & 1.2 & 39.2 & 40.4 \\
\hline $\mathcal{S}_{\mathcal{B}}$ & 16 & IBM_FV_2002_23 & 10320 & G & 8 & 8 & 7618 & 14.52 & 0.69 & 911.29 & 26.5 & 115.68 & 2.36 & 118.04 & 1 & 0.36 & 103.01 & 103.37 & - & 0.35 & 88.41 & 88.76 & 2 & 0.74 & 88.41 & 89.15 \\
\hline $\mathcal{S}_{\mathcal{B}}$ & 17 & IBM_FV_2002_27 & 4320 & G & 8 & 6 & 431223 & 3.09 & 4.54 & 415.98 & 23.61 & 24.43 & 25.01 & 49.44 & 0 & 1.77 & 49.44 & 51.21 & 0 & 1.78 & 49.44 & 51.22 & 0 & 24.5 & 49.44 & 73.94 \\
\hline $\mathcal{S}_{\mathcal{B}}$ & 18 & IBM_FV_2002_31_2 & 22720 & G & 17 & 17 & & 9.81 & 0.19 & & & 168.2 & 2.57 & 170.77 & 17 & 0.19 & & 0.19 & 17 & 0.19 & & 0.19 & 17 & 0.19 & 4 & 0.19 \\
\hline
\end{tabular}

